\title{
Translated simple modules for Lie algebras and simple supermodules for Lie superalgebras
}

\author{
Chih-Whi Chen $^{1,2} \cdot$ Kevin Coulembier ${ }^{3} \cdot$ Volodymyr Mazorchuk $^{1}$
}

Received: 8 October 2018 / Accepted: 11 March 2020 / Published online: 6 April 2020

(c) The Author(s) 2020

\begin{abstract}
We prove that the tensor product of a simple and a finite dimensional $\mathfrak{s l}_{n}$-module has finite type socle. This is applied to reduce classification of simple $\mathfrak{q}(n)$-supermodules to that of simple $\mathfrak{s l}_{n}$-modules. Rough structure of simple $\mathfrak{q}(n)$-supermodules, considered as $\mathfrak{s l}_{n}$-modules, is described in terms of the combinatorics of category $\mathcal{O}$.
\end{abstract}

Keywords Lie algebra $\cdot$ Simple module $\cdot$ Tensor product $\cdot$ Socle $\cdot$ Lie superalgebra Simple supermodule $\cdot$ Rough structure

Mathematics Subject Classification 17B10 $\cdot$ 17B55

\section{Introduction}

For a finite dimensional Lie algebra $\mathfrak{k}$, we consider modules of the form $S \otimes E$, where $S$ is a simple (but not necessarily finite dimensional) $\mathfrak{k}$-module and $E$ is a finite dimensional $\mathfrak{k}$-module. The module $S \otimes E$ is always noetherian and it is natural to ask, see e.g. [32, Section 1.3], whether $S \otimes E$ is artinian. The latter is true, for example, if $\mathfrak{k} \cong \mathfrak{s l}_{2}(\mathbb{C})$. However, in the general case the answer is negative, see $\left[42\right.$, Theorem 4.1] for $\mathfrak{k}=\mathfrak{s l}_{2}(\mathbb{C}) \oplus \mathfrak{s l}_{2}(\mathbb{C})$.

In the first half of the present paper, we investigate the following two questions.

Q1: Does the quotient of $S \otimes E$ by its radical have finite length?

Q2: Is the socle of $S \otimes E$ is an essential submodule?

\footnotetext{
$凶$ Chih-Whi Chen

cwchen@math.ncu.edu.tw

Kevin Coulembier

kevin.coulembier@sydney.edu.au

Volodymyr Mazorchuk

mazor@math.uu.se

1 Department of Mathematics, Uppsala University, Box 480, 75106 Uppsala, Sweden

2 Present Address: Department of Mathematics, National Central University, Zhongli District, Taoyuan City 32001, Taiwan

3 School of Mathematics and Statistics, University of Sydney, Sydney, NSW 2006, Australia
} 
Note that, since the module $S \otimes E$ is noetherian, its socle must have finite length and its radical must be superfluous. We show that question Q1 has an affirmative answer for arbitrary Lie algebras over arbitrary fields. We also show that Q2 has an affirmative answer for reductive Lie superalgebras over $\mathbb{C}$ of type $A$. For the latter we apply the theory of projective functors of [3] and some specifics about Kazhdan-Lusztig combinatorics in type $A$.

Our interest in Q2 stems from an application to representation theory of Lie superalgebras, which occupies the second half of the paper. Let $\mathfrak{g}$ be a finite dimensional Lie superalgebra and $\mathfrak{g}_{0}$ be the Lie algebra forming the even part of $\mathfrak{g}$. A basic problem in the representation theory of $\mathfrak{g}$ is classification of simple $\mathfrak{g}$-supermodules. This problem is, most probably, too difficult in the general case. However, a natural variant of this problem is reduction to classification of simple $\mathfrak{g}_{0}$-modules. In [10] it was shown that for type I Lie superalgebras there is a natural bijection between simple $\mathfrak{g}$-supermodules and simple $\mathfrak{g}_{0}$-supermodules (i.e. pairs consisting of a simple $\mathfrak{g}_{0}$-module and an element in $\{0,1\}$ ). Such a nice result seems unrealistic outside type I. However, in the present paper we provide a weaker result (see Theorem 47) for all finite dimensional classical complex Lie superalgebras for which $\mathfrak{g}_{0}$ has type $A$. The crucial ingredient in the proof is the fact that any $\mathfrak{g}$-supermodule is a quotient of an induced $\mathfrak{g}_{0}^{-}$ module. And this latter fact follows from the fact that, in case $\mathfrak{g}_{0}$ has type $A$, question Q2 has an affirmative answer (see Proposition 22 in the first half of the paper).

The paper is organized as follows: Sect. 2 collects all necessary preliminaries for the first half of the paper. Section 3 studies socles and radicals in the biggest possible generality. Section 4 concentrates on similar questions for semisimple Lie algebras over $\mathbb{C}$. Section 5 deals with the very specific case of a sum of copies of $\mathfrak{s h}_{2}$. In Sect. 6 we collected necessary preliminaries about Lie superalgebras. Section 7 is devoted to classification of simple supermodules for Lie superalgebras with type $A$ even part and to the study of rough structure of such supermodules. Finally, Sect. 8 describes behavior of Kac induction functor, with respect to socles and radicals, for Lie superalgebras of type I.

\section{Preliminaries on socles and radicals}

We denote by $\mathbb{N}$ the set of all non-negative integers and by $\mathbb{Z}_{>0}$ the set of all positive integers.

\subsection{Socle, radical and multiplicities}

Let $R$ be a unital ring and $M$ a left $R$-module. A submodule $N \subset M$ is essential if $K \cap N=0$ implies $K=0$, for all submodules $K \subset M$. A submodule $N \subset M$ is superfluous if $K+N=M$ implies $K=M$, for all submodules $K \subset M$.

The socle $\operatorname{soc}(M)$ is defined as the sum of all simple submodules of $M$, in case $M$ has a simple submodule, and as zero otherwise. Equivalently, $\operatorname{soc}(M)$ is the maximal semisimple submodule of $M$.

Definition 1 We will say that an $R$-module $M$ has finite type socle if

(a) $\operatorname{soc}(M)$ has finite length (and hence is a finite direct sum of simple modules);

(b) $\operatorname{soc}(M)$ is an essential submodule of $M$.

Remark 2 (i) Sometimes modules with finite type socle are called finitely cogenerated modules. We do not use this terminology due to lack of symmetry with finitely generated modules, see Remark 5(i). 
(ii) If $\operatorname{soc}(M)$ has finite length, then $M$ has finite type socle if and only if all non-zero submodules of $M$ have non-zero socle.

(iii) If $M$ is noetherian, then $\operatorname{soc}(M)$ has finite length.

The radical $\operatorname{rad}(M)$ is the intersection of all maximal submodules of $M$. By convention, we have $\operatorname{rad}(M)=M$ in case $M$ has no maximal submodules.

Remark 3 Equivalently, $\operatorname{rad}(M)$ is the sum of all superfluous submodules. Indeed, any superfluous submodule is, clearly, contained in any maximal submodule. Now, assume that there is $x \in \operatorname{rad}(M)$ which is not contained in the sum of all superfluous submodules. Then $R x$ is not superfluous and hence $R x+N=M$, for some proper submodule $N$. By Zorn's lemma, without loss of generality, we may assume that $N$ is maximal (with respect to inclusions) among all submodules of $M$ that do not contain $x$. As $x \notin N$ and $R x+N=M$, we obtain that $N$ is, in fact, a maximal submodule of $M$. This now leads to a contradiction with $x \in \operatorname{rad}(M) \subset N$.

Definition 4 We will say that an $R$-module $M$ has finite type radical if

(a) $M / \operatorname{rad}(M)$ has finite length;

(b) $\operatorname{rad}(M)$ is a superfluous submodule of $M$.

Remark 5 (i) If $M$ has finite type radical, it follows easily that $M$ is finitely generated. In general, being finitely generated (or even noetherian) is not a sufficient condition for $M$ to have finite type radical, see Example 6.

(ii) If $M / \operatorname{rad}(M)$ has finite length and $M$ is finitely generated, then $M$ has finite type radical. This can be quickly reduced to the observation that any finitely generated module $N$ satisfies $\operatorname{rad}(N) \neq N$. Note that it is easy to construct examples of modules that are not finitely generated but whose quotient over their radical has finite length. For example, take the direct sum of a simple and an indecomposable injective module over the polynomial ring in one variable.

(iii) If $M$ is noetherian, then $\operatorname{rad}(M)$ is superfluous. This follows easily from Remark 3.

(iv) If $M / \operatorname{rad}(M)$ has finite length, then $M / \operatorname{rad}(M)$ is semisimple. This follows from the canonical morphism $M / \operatorname{rad}(M) \hookrightarrow \Pi_{\mathrm{m}} M / \mathrm{m}$, where $\mathrm{m}$ ranges over all maximal submodules of $M$. In this case we write $\operatorname{top}(M):=M / \operatorname{rad}(M)$. If $M / \operatorname{rad}(M)$ has infinite length, then $M / \operatorname{rad}(M)$ does not have to be semisimple, see Example 6 .

Example 6 Consider $R=\mathbb{C}[x]$, with $M=R$ being the (finitely generated) left regular $R$ module. For $\lambda \in \mathbb{C}$, let $(x-\lambda)$ denote the ideal in $\mathbb{C}[x]$ generated by the element $x-\lambda$. Then we have

$$
\operatorname{rad}(M)=\bigcap_{\lambda \in \mathbb{C}}(x-\lambda)=0 .
$$

Since $M / \operatorname{rad}(M)$ does not have finite length, $M$ does not have finite type radical.

For an $R$-module $M$, consider a filtration $F_{\bullet} M$ of $M$ of length $p \in \mathbb{N}$ :

$$
0=F_{p} M \subset \cdots \subset F_{i+1} M \subset F_{i} M \subset \cdots \subset F_{0} M=M .
$$

For a short exact sequence

$$
0 \rightarrow M_{1} \rightarrow M \rightarrow M_{2} \rightarrow 0
$$


we can define $F_{i} M_{1}=M_{1} \cap F_{i} M$ and $F_{i} M_{2}=\left(F_{i} M+M_{1}\right) / M_{1}$. These yield filtrations $F_{\bullet} M_{1}$ and $F_{\bullet} M_{2}$ of $M_{1}$ and $M_{2}$, and short exact sequences

$$
0 \rightarrow F_{i} M_{1} \rightarrow F_{i} M \rightarrow F_{i} M_{2} \rightarrow 0, \text { for all } 0 \leq i \leq p .
$$

For a simple $R$-module $L$, the multiplicity $[M: L] \in \mathbb{N} \cup\{\infty\}$ of $L$ in $M$ is

$$
[M: L]=\sup _{F_{\bullet}}\left|\left\{i \mid F_{i} M / F_{i+1} M \cong L\right\}\right|,
$$

where $F_{\bullet}$ ranges over all finite filtrations of $M$. By the above, for a short exact sequence (2.1), we have

$$
[M: L]=\left[M_{1}: L\right]+\left[M_{2}: L\right]
$$

Lemma 7 Assume that $R$ is an algebra over $\mathbb{C}$ which is (at most) countably generated. If an $R$-module $M$ has finite type radical and simple top $S$, then

$$
\operatorname{dim}_{\mathbb{C}} \operatorname{Hom}_{R}(M, N) \leq[N: S],
$$

for all $R$-modules $N$.

Proof Since $M / \operatorname{rad}(M)$ is simple and $\operatorname{rad}(M)$ superfluous, it follows that every proper submodule of $M$ is contained in $\operatorname{rad}(M)$. Consequently, we have the inequality $[M / K: S] \geq 1$, for each proper submodule $K$. It also follows from the above (using Dixmier's version of Schur's lemma) that we have

$$
\operatorname{dim} \operatorname{Hom}_{R}(M, S)=1 \text {. }
$$

Furthermore, since a non-zero morphism $\alpha: M \rightarrow N$ yields a submodule $\operatorname{im}(\alpha)$ of $N$ with $[\operatorname{im}(\alpha): S] \geq 1$, we find that $[N: S]=0$ implies $\operatorname{Hom}_{R}(M, N)=0$.

Now assume that $n:=[N: S]<\infty$. By definition, $N$ admits a finite filtration $F_{\bullet} N$, such that each module $Q_{i}:=F_{i} N / F_{i-1} N$ satisfies $\left[Q_{i}: S\right]=0$ or $Q_{i} \cong S$. The latter option occurs exactly $n$ times. By left exactness of the Hom functor and the above paragraph we thus have

$$
\operatorname{dim} \operatorname{Hom}_{R}(M, N) \leq \sum_{i} \operatorname{dim} \operatorname{Hom}_{R}\left(M, Q_{i}\right)=n,
$$

which concludes the proof.

\subsection{Lie algebras}

Let $\mathbb{k}$ be a field and $\mathfrak{k}$ a finite dimensional Lie algebra over $\mathbb{k}$. The universal enveloping algebra of $\mathfrak{k}$ will be denoted by $U:=U(\mathfrak{k})$ and the center of $U$ by $Z=Z(\mathfrak{k})$. Denote by $\Theta$ the set of central characters $\chi: Z(\mathfrak{k}) \rightarrow \mathbb{C}$. We set $\mathrm{m}_{\chi}=\operatorname{ker} \chi$, for all $\chi \in \Theta$.

We denote by $U$-mod the abelian category of all finitely generated left $U$-modules. For $\chi \in \Theta$, denote by $U-\bmod _{\chi}$ the full subcategory of $U$-mod consisting of all modules on which the action of $m_{\chi}$ is locally nilpotent. Set

$$
U-\bmod _{Z}=\bigoplus_{\chi \in \Theta} U-\bmod _{\chi} .
$$


For each $\chi \in \Theta$, we denote by $\operatorname{Pr}_{\chi}$ the projection from $U-\bmod _{Z}$ to $U-\bmod _{\chi}$ with respect to the decomposition above.

Let $\mathcal{F}$ denote the category of all finite dimensional $U$-modules. We denote the duality $\operatorname{Hom}_{\mathbb{k}}(-, \mathbb{k})$ on $\mathcal{F}$ by $*$, where the left module structure is obtained using the antiautomorphism of $U$ given by $X \mapsto-X$, for $X \in \mathfrak{k}$.

For a finite dimensional module $E$, we have the corresponding exact endofunctor $F_{E}=$ $-\otimes E$ of $U$-mod. The functor $F_{E}$ is left and right adjoint to $F_{E^{*}}$, see e.g. [3, $\left.\S 2.1(\mathrm{~d})\right]$. In case of a semisimple algebra $\mathfrak{g}$, the direct summands of the restriction to $U-\bmod _{Z}$ of the functors $F_{E}$ are known as projective functors, see [3].

For any $M \in U$-mod, we denote by $\operatorname{Ann}(M)$ the two-sided ideal in $U$ that consists of all elements which annihilate every vector in $M$. The arguments in [27, Kapitel 5] show that, for any $M_{1}, M_{2} \in U$-mod, $E \in \mathcal{F}$ and $\chi \in \Theta$, we have

$$
\operatorname{Ann}\left(M_{1}\right) \subset \operatorname{Ann}\left(M_{2}\right) \Rightarrow \operatorname{Ann}\left(\operatorname{Pr}_{\chi}\left(M_{1} \otimes E\right)\right) \subset \operatorname{Ann}\left(\operatorname{Pr}_{\chi}\left(M_{2} \otimes E\right)\right) .
$$

\subsection{Gelfand-Kirillov dimension and Bernstein number}

We fix an arbitrary field $\mathbb{k}$ and a finite dimensional Lie algebra $\mathfrak{k}$ over $\mathbb{k}$. Consider the filtration

$$
U=\bigcup_{n \in \mathbb{N}} U_{n}
$$

where $U_{n}$ is spanned, as a vector space, by all products of $n$ or fewer elements of $\mathfrak{k}$. By the PBW theorem, the associated graded algebra $\operatorname{gr}(U)$ is isomorphic to the symmetric algebra $S(\mathfrak{k})$. This allows us to use an alternative definition of Gelfand-Kirillov dimension, see [33, Section 7], which we recall below.

For a (non-zero) finitely generated left $U$-module $M$, with generating subspace $M_{0}$, set $M_{n}=U_{n} M_{0}$, for all $n \in \mathbb{N}$. There exists $n_{0} \in \mathbb{N}$, such that we have $d \in \mathbb{N}$ and $\left\{a_{i} \in \mathbb{Z}, 0 \leq\right.$ $i \leq d\}$, with $a_{d} \neq 0$, for which

$$
\operatorname{dim}_{\mathbb{k}} M_{n}=\sum_{i=0}^{d} a_{i}\left(\begin{array}{l}
n \\
i
\end{array}\right), \quad \text { for all } n \geq n_{0} .
$$

The right-hand side is the Hilbert-Samuel polynomial in $n$. The degree of this polynomial is the Gelfand-Kirillov dimension $\operatorname{GK}(M):=d \in \mathbb{N}$ and the leading coefficient is the Bernstein number $\mathrm{e}(M):=a_{d} \in \mathbb{Z}_{>0}$. These two numbers do not depend on the choice of $M_{0}$.

By [27, Lemma 8.8], for any $M \in U-\bmod$ and $E \in \mathcal{F}$, we have

$$
\mathrm{GK}(M \otimes E)=\mathrm{GK}(M), \quad \text { and } \mathrm{e}(M \otimes E)=\operatorname{dim}_{\mathbb{k}}(E) \mathrm{e}(M) .
$$

The following statement can be found in [33, Theorem 7.7].

Lemma 8 Let $0 \rightarrow M_{1} \rightarrow M \rightarrow M_{2} \rightarrow 0$ be a short exact sequence in $U$-mod.

(i) We have $G K(M)=\max \left\{G K\left(M_{1}\right), G K\left(M_{2}\right)\right\}$.

(ii) If $G K\left(M_{1}\right)=G K(M)=G K\left(M_{2}\right)$, then we have $e(M)=e\left(M_{1}\right)+e\left(M_{2}\right)$.

Definition 9 A finite filtration $F_{\bullet} M$ of $M \in U$-mod is $G K$-complete if

$$
\mathrm{GK}\left(F_{i} M / F_{i+1} M\right)=\mathrm{GK}(M) \text { implies } F_{i} M / F_{i+1} M \text { is simple, for all } i \text {. }
$$

By the discussion at the end of Sect. 2.1, we have the following observation. 
Lemma 10 Assume that $M \in U$-mod admits a $G K$-complete filtration. If $N$ is a subquotient of $M$ with the same Gelfand-Kirillov dimension, then $N$ admits a GK-complete filtration as well.

\subsection{Triangular decomposition}

In this section we assume that $\mathfrak{g}$ is a semisimple Lie algebra over $\mathbb{C}$. In order to recall the classification of projective functors for $\mathfrak{g}$ from [3], it is convenient to choose a triangular decomposition

$$
\mathfrak{g}=\mathfrak{n}^{-} \oplus \mathfrak{h} \oplus \mathfrak{n}^{+}
$$

of $\mathfrak{g}$ with $\mathfrak{h}$ a Cartan subalgebra and $\mathfrak{b}=\mathfrak{h} \oplus \mathfrak{n}^{+}$a Borel subalgebra. Denote by $\Phi \subset \mathfrak{h}^{*}$ the set of roots of $\mathfrak{g}$ with respect to $\mathfrak{h}$. We have $\Phi=\Phi^{+} \sqcup \Phi^{-}$, with $\Phi^{+}=-\Phi^{-}$being the roots of $\mathfrak{n}^{+}$. For each root $\alpha \in \Phi$, we have the coroot $h_{\alpha} \in \mathfrak{h}$.

Consider the Weyl group $W=W(\mathfrak{g}: \mathfrak{h})$ with its defining action on $\mathfrak{h}^{*}$. The group $W$ is generated by $s_{\alpha}$, where $\alpha \in \Phi$, and the action of these generators on $\mathfrak{h}^{*}$ is given by $s_{\alpha}(\lambda)=\lambda-\lambda\left(h_{\alpha}\right) \alpha$, where $\lambda \in \mathfrak{h}^{*}$. For each $\lambda \in \mathfrak{h}^{*}$, we denote the stabilizer of $\lambda$ in $W$ by $W_{\lambda}:=\{w \in W \mid w(\lambda)=\lambda\}$. We have the set of integral weights

$$
\Lambda:=\left\{\lambda \in \mathfrak{h}^{*} \mid \lambda\left(h_{\alpha}\right) \in \mathbb{Z}, \text { for all } \alpha \in \Phi\right\} .
$$

For each $E \in \mathcal{F}$, we denote by $\operatorname{supp}(E) \subset \Lambda$ the support of $E$, that is the set of all $\mathfrak{h}$-weights of $E$. Then we have

$$
\Lambda=\bigcup_{E \in \mathcal{F}} \operatorname{supp}(E)
$$

For a coset $\Gamma \in \mathfrak{h}^{*} / \Lambda$, we have the integral Weyl group $W^{\Gamma} \subset W$, which is generated by all reflections corresponding to $\alpha \in \Phi$ for which $\lambda\left(h_{\alpha}\right) \in \mathbb{Z}$, where $\lambda \in \Gamma$.

We have the Harish-Chandra isomorphism $\eta^{*}: Z(\mathfrak{g}) \stackrel{\sim}{\rightarrow} S(\mathfrak{h})^{W}$ and an epimorphism

$$
\eta: \mathfrak{h}^{*} \rightarrow \Theta, \text { where } \eta(\lambda)\left(_{-}\right)=\eta^{*}\left(_{-}\right)(\lambda) \text {, for all } \lambda \in \mathfrak{h}^{*} .
$$

The fibers of $\eta$ are precisely the Weyl group orbits in $\mathfrak{h}^{*}$. We call a central character $\chi \in \Theta$ regular if the set $\eta^{-1}(\chi)$ has size $|W|$. We call $\chi$ integral if $\eta^{-1}(\chi) \subset \Lambda$.

We introduce the partial order $\leq$ on $\mathfrak{h}^{*}$ which is generated by $\mu \leq \lambda$, if $\mu=s_{\alpha} \lambda$, for some $\alpha \in \Phi^{+}$with $\lambda\left(h_{\alpha}\right) \in \mathbb{Z}_{\geq 0}$. Hence, $\lambda \in \mathfrak{h}^{*}$ is dominant (maximal) if $\lambda\left(h_{\alpha}\right) \notin \mathbb{Z}_{<0}$, for all $\alpha \in \Phi^{+}$.

Associated to the triangular decomposition above, we have the BGG category $\mathcal{O}$ defined as the full subcategory of $U$-mod consisting of all weight modules which are locally $U\left(\mathfrak{n}^{+}\right)$finite, see $[4,24]$. We denote by $\rho$ the half of the sum of all elements of $\Phi^{+}$. For $\lambda \in \mathfrak{h}^{*}$, we have the Verma module $\Delta_{\lambda}=U \otimes_{U(\mathfrak{b})} \mathbb{C}_{\lambda-\rho}$, induced from the one-dimensional module $\mathbb{C}_{\lambda-\rho}$ of $\mathfrak{b}$ on which $\mathfrak{h}$ acts through $\lambda-\rho$. The unique simple quotient of $\Delta_{\lambda}$ is denoted by $L_{\lambda}$ and the projective cover of $L_{\lambda}$ in $\mathcal{O}$ is denoted by $P_{\lambda}$.

\subsection{Projective functors for semisimple Lie algebras}

We keep the notation and assumptions of the previous subsection. Following [3, Section 1.4], we have the set

$$
\Xi^{0}=\{(\mu, \lambda) \mid \lambda-\mu \in \Lambda\} \subset \mathfrak{h}^{*} \times \mathfrak{h}^{*}
$$


We set $\Xi=\Xi^{0} / W$, for the diagonal action of $W$ on $\mathfrak{h}^{*} \times \mathfrak{h}^{*}$. Each class in $\Xi$ contains at least one $(\mu, \lambda)$, where $\lambda$ is dominant and $\mu$ is such that $\mu \leq w \mu$, for all $w \in W_{\lambda}$. We call such a pair a proper representative. The following claim can be found in [3, Theorem 3.3].

Lemma 11 Each projective functor on $U-\bmod _{Z}$ decomposes into a finite direct sum of indecomposable projective functors. We have a bijection $\xi \mapsto F(\xi)$ between $\Xi$ and the set of isomorphism classes of indecomposable projective functors as follows. For each proper representative $(\mu, \lambda)$ of $\xi \in \Xi$, the functor $F_{\lambda}^{\mu}=F(\xi)$ satisfies

$$
F_{\lambda}^{\mu}: U-\bmod _{\eta(\lambda)} \rightarrow U-\bmod _{\eta(\mu)}, \text { with } F_{\lambda}^{\mu}\left(\Delta_{\lambda}\right) \cong P_{\mu} .
$$

Note that the above lemma implies that the decomposition into indecomposable projective functors is, in fact, unique, up to isomorphism. In case we work with non-integral central characters, we have several ways of denoting the same indecomposable projective functor. The first claim of the following lemma can be found in [3, Theorem 4.1] while the second claim can be found in [27, § 4.13].

Lemma 12 Consider $\lambda, \mu \in \mathfrak{h}^{*}$ dominant with $\lambda-\mu \in \Lambda$.

(i) If $W_{\lambda}=W_{\mu}$, then we have an equivalence of categories

$$
F_{\lambda}^{\mu}: U-\bmod _{\eta(\lambda)} \stackrel{\sim}{\rightarrow} U-\bmod _{\eta(\mu)} .
$$

(ii) If $W_{\lambda}=\{e\}$, then we take the longest element $w_{0}^{\mu}$ of $W_{\mu}$ and set $\lambda^{\prime}=w_{0}^{\mu} \lambda$. The functors $\left(F_{\mu}^{\lambda^{\prime}}, F_{\lambda}^{\mu}\right)$ are biadjoint, moreover, $F_{\lambda}^{\mu} F_{\mu}^{\lambda^{\prime}} \cong \operatorname{Id}_{\eta(\mu)}^{\oplus\left|W_{\mu}\right|}$.

Denote by $U-\bmod _{\chi}^{0}$ the full subcategory of $U-\bmod _{\chi}$ consisting of all modules $M$ with $\mathrm{m}_{\chi} \subset \operatorname{Ann}(M)$. The following claim can be found in [3, Theorem 3.5].

Lemma 13 Let $\lambda \in \mathfrak{h}^{*}$ be dominant and $\left(\lambda, \mu_{1}\right)$ and $\left(\lambda, \mu_{2}\right)$ be proper representatives. Evaluation yields an isomorphism

$$
\operatorname{Nat}\left(F_{\lambda}^{\mu_{1}}, F_{\lambda}^{\mu_{2}}\right) \stackrel{\sim}{\rightarrow} \operatorname{Hom}_{\mathfrak{g}}\left(F_{\lambda}^{\mu_{1}} \Delta_{\lambda}, F_{\lambda}^{\mu_{2}} \Delta_{\lambda}\right), \quad \alpha \mapsto \alpha_{\Delta_{\lambda}},
$$

where $\operatorname{Nat}\left(F_{\lambda}^{\mu_{1}}, F_{\lambda}^{\mu_{2}}\right)$ stands for the space of all natural transformations from $F_{\lambda}^{\mu_{1}}$ to $F_{\lambda}^{\mu_{2}}$ as functors $U-\bmod _{\eta(\lambda)}^{0} \rightarrow U-\bmod _{\eta\left(\mu_{i}\right)}$.

\subsection{Kazhdan-Lusztig combinatorics and type A}

We keep the notation and assumptions of the previous subsection.

For a fixed regular dominant $\lambda \in \Lambda$, we use the notation $\theta_{x}:=F_{\lambda}^{x \lambda}$, for all $x \in W$. It then follows from the validity of the Kazhdan-Lusztig conjecture, see [1,8] and Lemma 11, that the composition of the projective functors $\theta_{x}$ is governed by Kazhdan-Lusztig combinatorics. Concretely, if we have the Kazhdan-Lusztig basis $\left\{C_{w}^{\prime} \mid w \in W\right\}$ of the group ring $\mathbb{Z} W$ (i.e. the Hecke algebra of $W$ specialized at $q=1$ ) of $[29, \S 1]$, then we have

$$
C_{x}^{\prime} C_{y}^{\prime}=\sum_{z \in W} h_{x, y, z} C_{z}^{\prime} \text { and } \theta_{y} \theta_{x}=\bigoplus_{z \in W} \theta_{z}^{\oplus h_{x, y, z}},
$$

for the same coefficients $h_{x, y, z} \in \mathbb{Z}_{\geq 0}$, see [25, Corollary 5.2.4].

Consequently, the Kazhdan-Lusztig preorders of [29, § 1] can be realised by projective functors on a regular block. For convenience, we extend this to the entire category of projective 
functors. We thus introduce the following preorder $\preceq$ on the set of (isomorphism classes of) indecomposable projective functors. We say that $F \preceq G$ if $G$ appears as a direct summand of $F^{\prime} \circ F \circ F^{\prime \prime}$, for some projective functors $F^{\prime}, F^{\prime \prime}$. We have the corresponding equivalence relation, denoted $F \sim G$, which means that $F \preceq G \preceq F$. Equivalence classes for $\sim$ are called two-sided cells. Similarly one defines the left and the right preorders and the left and the right cells.

If the Weyl group $W$ is the symmetric group, the preorder $\preceq$ can be described in terms of the dominance order on partitions using the Robinson-Schensted correspondence, see [20, Theorem 5.1]. As a consequence, each two-sided Kazhdan-Lusztig cell contains the longest element of some parabolic subgroup. Another consequence (see [20, Corollary 5.6]) is that a left and a right cell inside the same two-sided cell intersect in at most one element. By the above and [20, Theorem 5.3] this translates into two well-known facts for Lie algebras of type A.

Let $\lambda \in \mathfrak{h}^{*}$ be integral, regular and dominant, and $\theta$ an indecomposable projective endofunctor of $U-\bmod _{\eta(\lambda)}$.

Fact 1: There exists $x \in W$, which is the longest element of a parabolic subgroup such that $\theta \sim \theta_{x}$.

Fact 2: The only indecomposable projective endofunctor $\theta^{\prime}$ of $U-\bmod _{\eta(\lambda)}$ which satisfies $\theta^{\prime} \sim \theta$ and appears both as a direct summand in $\theta \circ G_{1}$ and $G_{2} \circ \theta$, for some projective functors $G_{1}, G_{2}$, is $\theta$ itself.

We translate these facts into the formulation that we will require.

Lemma 14 Assume $\mathfrak{g}$ is of type A and fix a -equivalence class of projective functors.

(i) The class contains $F_{\mu}^{\mu}=\operatorname{Id}_{\eta(\mu)}$, the identity functor of $U$ - $\bmod _{\eta(\mu)}$, for some dominant $\mu \in \mathfrak{h}^{*}$.

(ii) No other indecomposable projective endofunctors of $U-\bmod _{\eta(\mu)}$ are contained in the class.

Proof We start from an arbitrary indecomposable projective functor

$$
F: U-\bmod _{\chi_{1}} \rightarrow U-\bmod _{\chi_{2}}
$$

and consider its equivalence class. It follows from Lemma 12(ii) that $F \sim G$, for some endofunctor $G$ of $U-\bmod _{\eta(\lambda)}$, where $\lambda$ is any fixed dominant regular weight in $\eta^{-1}\left(\chi_{1}\right)+\Lambda$.

Assume, for simplicity, that $\lambda$ is integral. The non-integral case is proved using the same arguments, since the integral Weyl group in type $A$ is always of type $A$. By Fact 1 , we have $G \sim \theta_{x_{0}}$, for the longest element $x_{0}$ of some parabolic subgroup of $W$. Take some dominant $\mu \in \Lambda$ such that $W_{\mu}$ is this parabolic subgroup. Then we have $\theta_{x_{0}}=F_{\mu}^{\lambda^{\prime}} F_{\lambda}^{\mu}$. By Lemma 12(ii), we have

$$
\theta_{x_{0}} \sim F_{\mu}^{\lambda^{\prime}} \sim F_{\lambda}^{\mu} \sim \operatorname{Id}_{\eta(\mu)} .
$$

Consequently, we have $F \sim \operatorname{Id}_{\eta(\mu)}$, which concludes the proof of part (i).

To prove part (ii), we assume we have $\operatorname{Id}_{\eta(\mu)} \sim H$, for some indecomposable projective endofunctor $H$ of $U-\bmod _{\eta(\mu)}$. We set $H_{1}:=F_{\mu}^{\lambda^{\prime}} H F_{\lambda}^{\mu}$. Lemma 12(ii) implies that

$$
\theta_{x_{0}} H_{1}=H_{1}^{\oplus\left|W_{\mu}\right|}=H_{1} \theta_{x_{0}} \text { and } F_{\lambda}^{\mu} H_{1} F_{\mu}^{\lambda^{\prime}}=H^{\oplus\left|W_{\mu}\right|^{2}} .
$$

The second equation shows that there exists an indecomposable summand $H_{1}^{\prime}$ of $H_{1}$ for which we have $H_{1}^{\prime} \sim H \sim \theta_{x_{0}}$. By Fact 2, the first equation therefore implies that $H_{1}^{\prime}=\theta_{x_{0}}$. 
Applying the second equation again together with Lemma 12(ii) then shows that $\operatorname{Id}_{\eta(\mu)}$ must appear as a direct summand in $H^{\oplus\left|W_{\mu}\right|^{2}}$. This is only possible if $H=\operatorname{Id}_{\eta(\mu)}$. This concludes the proof of part (ii).

Remark 15 Facts 1 and 2 generally fail for other Weyl groups, implying that Lemma 14 is specific to Lie algebras of type $A$. For instance, Fact 1 fails for $B_{5}$, since in this case the unique two-sided cell having the a-value 11 contains no longest element of parabolic subgroups (a private communication by Tobias Kildetoft). Fact 1 remains valid, for instance, for $\left\{B_{n} \mid n<5\right\}$, but there Fact 2 fails, for $n>1$, see [34, Appendix].

\section{Arbitrary Lie algebras}

Fix a finite dimensional Lie algebra $\mathfrak{k}$ over a field $\mathbb{k}$ and set $U=U(\mathfrak{k})$.

\subsection{Connection with GK dimension}

Theorem 16 Fix a simple $U$-module $S$ and $E \in \mathcal{F}$. Then $T:=S \otimes E$ has finite type radical, and $\operatorname{soc}(T)$ has finite length. Furthermore, the following are equivalent:

(a) The module $T=S \otimes E$ has finite type socle.

(b) Any $N \in U$-mod with non-zero morphism $N \otimes E^{*} \rightarrow S$ has a simple subquotient $L$ with non-zero morphism $L \otimes E^{*} \rightarrow S$.

(c) Every non-zero submodule of $T$ contains a simple subquotient of Gelfand-Kirillov dimension $G K(S)$.

We start the proof with the following lemma.

Lemma 17 With $T$ as in Theorem 16, for any short exact sequence of $U$-modules

$$
0 \rightarrow M_{1} \rightarrow T \rightarrow M_{2} \rightarrow 0, \text { with } M_{1} \neq 0 \neq M_{2},
$$

we have $G K\left(M_{1}\right)=G K(S)=G K\left(M_{2}\right)$.

Proof We prove that $\mathrm{GK}\left(M_{1}\right)=\mathrm{GK}(S)$. The statement for $M_{2}$ is proved similarly. By Lemma 8(i) and Eq. (2.3), we have $\operatorname{GK}\left(M_{1}\right) \leq \mathrm{GK}(S)$. By adjunction

$$
0 \neq \operatorname{Hom}_{\mathfrak{k}}\left(M_{1}, T\right) \cong \operatorname{Hom}_{\mathfrak{k}}\left(M_{1} \otimes E^{*}, S\right) .
$$

Hence, $S$ is a quotient of $M_{1} \otimes E^{*}$. Lemma 8(i) and Eq. (2.3) thus imply that $\mathrm{GK}(S) \leq \mathrm{GK}\left(M_{1}\right)$, which concludes the proof.

Lemma 18 There exists $k \in \mathbb{Z}_{\geq 0}$, such that $T$ cannot have a semisimple submodule or quotient of length greater than $k$.

Proof We prove the claim for quotients, the case of submodules is proved similarly or follows from Remark 2(iii). By Lemma 17, a semisimple quotient of $T$ is a direct sum of simple modules with the GK dimension of each simple equal to $\operatorname{GK}(S)$. By Lemma 8(ii), we can thus choose $k=\mathrm{e}(T)$.

Corollary 19 The module $T$ has finite type radical. 
Proof Since $T$ is finitely generated, it suffices by Remark 5(ii) to show that the module $T / \operatorname{rad}(T)$ has finite length. If it would have infinite length, we could take an arbitrarily large, but finite, direct sum of simple modules as a quotient of $T$, which is contradicted by Lemma 18.

Proof of Theorem 16 This first claim is Corollary 19 and Lemma 18. Now we prove the equivalence of (a), (b) and (c).

First we prove that (a) implies (b). Take $N$ with non-zero $N \otimes E^{*} \rightarrow S$ and consider the corresponding non-zero morphism $\alpha: N \rightarrow S \otimes E$. The image $\operatorname{im}(\alpha)$ is a submodule of $S \otimes E$ and thus has a non-zero socle by assumption. We take a simple module $L$ in that socle, which, by construction, is a subquotient of $N$. The inclusion $L \hookrightarrow S \otimes E$ yields a non-zero morphism $L \otimes E^{*} \rightarrow S$.

Next we note that (b) trivially implies (c). So, it remains to show that (c) implies (a). We assume (c) holds and set $d:=\operatorname{GK}(S)$. To obtain a contradiction via Remark 2(ii), we assume we have a non-zero submodule $M$ of $T$ with zero socle. Assume we have $[M: L] \neq 0$, for some simple $U$-module $L$ with $\operatorname{GK}(L)=d$. We thus must have submodules

$$
M_{1} \subset M_{2} \subset M, \text { with } M_{2} / M_{1} \cong L .
$$

Since $M$ has zero socle, we have $M_{1} \neq 0$. By construction, $M_{1}$ is again a submodule of $T$ with zero socle. By Lemma 17 , we have $\mathrm{GK}\left(M_{1}\right)=d=\mathrm{GK}(M)$. By Lemma 8(ii), we have $\mathrm{e}(M)>\mathrm{e}\left(M_{1}\right)>0$. This means that, after repeating the above construction $M \mapsto M_{1}$ a finite number times, we obtain a non-zero submodule $N$ of $M$ (and hence of $T$ ) with zero socle, and such that $[N: L]=0$, for all simple $U$-modules with $\operatorname{GK}(L)=d$. It thus follows that any simple subquotient $L$ of $N$ has Gelfand-Kirillov dimension less than $d$. Hence assumption (c) yields a contradiction. This completes the proof.

Corollary 20 If $T=S \otimes E$ admits a $G K$-complete filtration, it has finite type socle.

Proof By Lemmata 10 and 17, any submodule of $T$ has a GK-complete filtration. Assume that $T$ does not have finite type socle. By Theorem 16, this means that $T$ has a submodule $N$ with $\operatorname{GK}(N)=d$ and such that $[N: L]=0$, for all simple modules $L$ with $\mathrm{GK}(L)=d$. Hence $N$ cannot have a GK-complete filtration, a contradiction.

\subsection{Restriction to blocks}

Theorem 21 Let $S$ be a simple $U$-module with central character $\chi \in \Theta$. If $F(S)$ has finite type socle, for each projective endofunctor $F$ of $U-\bmod _{\chi}$, then we have the following:

(i) The module $S \otimes E$ has finite type socle, for each $E \in \mathcal{F}$.

(ii) The module $S^{\prime} \otimes E$ has finite type socle, for each $E \in \mathcal{F}$ and simple submodule $S^{\prime}$ of $S \otimes V$, for some $V \in \mathcal{F}$.

Proof We start by proving Claim (i). We set $d=\operatorname{GK}(S)$ and use the equivalence between (a) and (c) in Theorem 16. We thus take an arbitrary submodule $N$ of $S \otimes E$. By adjunction, we have a non-zero morphism $N \otimes E^{*} \rightarrow S$, which implies $\operatorname{Pr}_{\chi}\left(N \otimes E^{*}\right)$ is not zero. Since $N \otimes E^{*}$ is a submodule of $S \otimes E \otimes E^{*}$, we have an inclusion

$$
\operatorname{Pr}_{\chi}\left(N \otimes E^{*}\right) \hookrightarrow \operatorname{Pr}_{\chi}\left(S \otimes E \otimes E^{*}\right) .
$$

By assumption, the socle of the right-hand side is an essential submodule. Hence we can take a simple submodule $L$ in the socle of the right hand side which is also contained in the 
left-hand side. Note that $\operatorname{GK}(L)=d$ by Lemma 17. So $L$ is a submodule of $N \otimes E^{*}$, which leads through adjunction to a non-zero morphism $L \otimes E \rightarrow N$. Since $L \otimes E$ has finite type radical, there is a simple submodule $L_{1}$ of $\operatorname{top}(L \otimes E)$ such that $\left[N: L_{1}\right] \neq 0$. By Lemma 17, we have $\operatorname{GK}\left(L_{1}\right)=d$. Claim (i) thus follows from Theorem 16.

Now we consider the set-up of claim (ii). By construction, $S^{\prime} \otimes E$ is a submodule of $S \otimes$ $V \otimes E$. By claim (i), the latter has finite type socle, completing the proof.

\subsection{Application to Lie superalgebras}

Let $\mathfrak{s}=\mathfrak{s}_{\overline{0}} \oplus \mathfrak{s}_{\overline{1}}$ be a finite dimensional Lie superalgebra over $\mathbb{k}$, see [40, Chapter 1]. The universal enveloping algebra $\widetilde{U}=U(\mathfrak{s})$ of $\mathfrak{s}$ is a $\mathbb{Z}_{2}$-graded associative algebra and a finite ring extension of $U=U\left(\mathfrak{s}_{\overline{0}}\right)$.

In the following we use the term simple $\widetilde{U}$-module to denote any of the following two notions

(I) A simple $\widetilde{U}$-module, without any reference to the $\mathbb{Z}_{2}$-grading;

(II) $\mathrm{A} \mathbb{Z}_{2}$-graded $\widetilde{U}$-module which has no proper graded submodules.

Proposition 22 Assume that $S \otimes E$ has finite type socle, for every simple $U$-module $S$ and any $E \in \mathcal{F}$. Then every simple $\widetilde{U}$-module is a quotient of a module of the form $\widetilde{U} \otimes_{U} L$, for some simple $U$-module $L$.

Proof Consider a simple $\widetilde{U}$-module $K$ in the sense of (I). In particular, it is generated by any vector in $K$. We denote by Res the restriction functor from $\widetilde{U}$-modules to $U$-modules, with left adjoint Ind and right adjoint Coind. $\operatorname{Since} \operatorname{Res}(K)$ is finitely generated, it is a noetherian $U$ module. Consequently, there is a simple $U$-module $S$ with non-zero morphism $\operatorname{Res}(K) \rightarrow S$. By adjunction, we have an inclusion $K \hookrightarrow$ Coind $S$. Applying the exact restriction functor gives

$$
\operatorname{Res}(K) \hookrightarrow S \otimes \Lambda\left(\mathfrak{s}_{\overline{1}}\right)^{*}
$$

Since the right-hand side has finite type socle, there is a simple $U$-module $L$, for which we have an inclusion $L \hookrightarrow \operatorname{Res}(K)$. Applying adjunction shows that we have a surjection Ind $L \rightarrow K$.

Consider a simple $\widetilde{U}$-module $K$ in the sense of (II). In particular, it is generated by any vector in $K_{\overline{0}} \cup K_{\overline{1}}$. We can follow the above procedure and make sure all relevant morphisms respect the $\mathbb{Z}_{2}$-grading. Any such morphism to or from a simple graded $\widetilde{U}$-module will then again automatically be surjective or injective, respectively. The claim follows.

\section{Semisimple Lie algebras over $\mathbb{C}$}

In this section, we work under the assumptions that $\mathbb{k}=\mathbb{C}$ and that the Lie algebra $\mathfrak{g}$ is semisimple.

\subsection{Type A}

Theorem 23 If $\mathfrak{g}$ is of type A, then every module $S \otimes E$, for $S$ simple and E finite dimensional, has finite type socle. 
Proof Let $S$ be an arbitrary simple module with central character $\chi$. Consider an indecomposable projective functor $F$ such that $F(S) \neq 0$ and for which every indecomposable projective functor $G \succeq F$ with $G(S) \neq 0$ satisfies $G \sim F$. Note that this is possible since we only have finite chains

$$
F \preceq F^{\prime} \preceq F^{\prime \prime} \preceq \cdots \quad \text { with } \quad F \nsim F^{\prime} \nsim F^{\prime \prime} \nsim \cdots .
$$

We consider the equivalence class generated by $F$ and take $\mu \in \mathfrak{h}^{*}$ as in Lemma 14 .

Since, by assumption, $F \sim \operatorname{Id}_{\eta(\mu)}$, we have projective functors

$$
F_{1}: U-\bmod _{\chi} \rightarrow U-\bmod _{\eta(\mu)} \quad \text { and } \quad F_{2}: U-\bmod _{\eta(\mu)} \rightarrow U-\bmod _{\chi}
$$

such that $F$ is a direct summand of $F_{2} \circ F_{1}=F_{2} \circ \operatorname{Id}_{\eta(\mu)} \circ F_{1}$.

Now we have that $F_{1} S$ is not zero. Since $F_{1} S$ has finite type radical, we can choose a simple quotient $L$ of $F_{1} S$. By adjunction, we find that $S$ is a submodule of $G_{1} L$, for $G_{1}$ the right adjoint of $F_{1}$.

By Theorem 21(ii), it thus suffices to prove that $H L$ has finite type socle, for each projective endofunctor $H$ of $U-\bmod _{\eta(\mu)}$. We claim that $H L=0$ for every indecomposable projective endofunctor $H$ different from the identity, which would thus complete the proof. Indeed, we even have $H F_{1} S=0$, since the statement in Lemma 14(ii),

$$
H \succeq \operatorname{Id}_{\eta(\mu)} \sim F \quad \text { with } \quad H \nsim \operatorname{Id}_{\eta(\mu)} \sim F
$$

implies that $H^{\prime} \succeq F$ with $H^{\prime} \nsim F$, for any indecomposable direct summand $H^{\prime}$ of $H F_{1}$.

\subsection{Low ranks in Type B and C}

Theorem 24 For $\mathfrak{g}=B_{n}=\mathfrak{s o}_{2 n+1}$ or $\mathfrak{g}=C_{n}=\mathfrak{s p}_{2 n}$, with $1 \leq n \leq 4$, every module $S \otimes E$, for $S$ simple with integral central character and $E$ finite dimensional, has finite type socle.

Proof For $n=1$, the Lie algebras are of type $A$. Now we focus on $n \in\{2,3,4\}$. In these cases, Fact 1 of Sect. 2.6 remains true, see [34, Appendix], where elements $x_{0}$ are printed in bold font. Consequently, Lemma 14(i) remains valid. Note that [34, Appendix] only discusses $B_{3}$ and $B_{4}$. However, since the Weyl groups of $B_{n}$ and $C_{n}$ are isomorphic, the latter is also included. Furthermore, the case for $B_{2}$ can be computed immediately by hand.

Lemma 14(ii) is no longer true as stated. However, one can calculate from [34, Appendix] that, in those cases where we have some indecomposable projective endofunctor $F$ of $U-\bmod _{\eta(\mu)}$ different from $\operatorname{Id}_{\eta(\mu)}$ but with $F \sim \operatorname{Id}_{\eta(\mu)}$, this $F$ is unique, up to isomorphism. Furthermore, we have $F \circ F \cong \operatorname{Id}_{\eta(\mu)} \oplus G$, where $G \nsim \operatorname{Id}_{\eta(\mu)}$, see the proof of [34, Theorem 31].

As in the proof of Theorem 23 it suffices to prove the following claim. Let $L$ be a simple module in $U-\bmod _{\eta(\mu)}$ which is annihilated by all projective endofunctors which are not in the equivalence class of $\operatorname{Id}_{\eta(\mu)}$, then $H L$ has finite type socle for each projective endofunctor $H$ of $U-\bmod _{\eta(\mu)}$. In those cases where $\operatorname{Id}_{\eta(\mu)}$ is alone in its class there is nothing to prove. So we assume that there exists a second functor $F$ in the class as in the above paragraph. However, in this case $F^{2}$ acts as the identity on simple modules which are annihilated by indecomposable projective endofunctors of $U-\bmod _{\eta(\mu)}$ not in the class of $\operatorname{Id}_{\eta(\mu)}$ (in fact, $F$ is an equivalence between appropriate subcategories of modules). Consequently $F L$ is a simple module. 
Remark 25 We can use the same arguments as in the proof of Theorem 24 for semisimple Lie algebras $\mathfrak{g}=\mathfrak{k} \oplus \mathfrak{l}$, with $\mathfrak{k}$ of type $A$ and $\mathfrak{l}$ of type $B_{n}$ or $C_{n}$, for $1 \leq n \leq 4$. Note that the arguments fail when we consider $\mathfrak{k} \oplus \mathfrak{l}$, with both $\mathfrak{k}$ and $\mathfrak{l}$ of low rank type $B$ and $C$.

\subsection{Regular central character reduction for semisimple Lie algebras}

Now we return to arbitrary semisimple Lie algebras over $\mathbb{C}$. We will show that for Q2, it will suffice to consider simple modules with regular central character.

Theorem 26 Take central characters $\chi_{r}$ and $\chi_{s}$ with $\chi_{r}$ regular and $\chi_{s}$ singular. Take dominant $\lambda \in \eta^{-1}\left(\chi_{r}\right)$ and $\mu \in \eta^{-1}\left(\chi_{s}\right)$ and assume $\lambda-\mu \in \Lambda$. Set $\theta^{\text {on }}=F_{\lambda}^{\mu}$ and $\theta^{\text {out }}=F_{\mu}^{\lambda^{\prime}}$, with $\lambda^{\prime}=w_{0}^{\mu} \lambda$, and set $n=\left|W_{\mu}\right|$.

Denote by $\operatorname{sim}_{r}$ and $\operatorname{sim}_{s}$ the set of isomorphism classes of simple modules with central character $\chi_{r}$ or $\chi_{s}$, respectively, and set

$$
\operatorname{sim}_{r}^{s}=\left\{S \in \operatorname{sim}_{r} \mid \theta^{\text {on }} S \neq 0\right\} .
$$

(i) We have a bijection

$$
t: \operatorname{sim}_{r}^{s} \stackrel{\sim}{\rightarrow} \operatorname{sim}_{s}, \quad S \mapsto \theta^{\text {on }} S, \quad \text { for } S \in \operatorname{sim}_{r}^{s},
$$

with inverse

$$
L \mapsto \operatorname{soc}\left(\theta^{\text {out }} L\right) \cong \operatorname{top}\left(\theta^{\text {out }} L\right), \text { for } L \in \operatorname{sim}_{s} .
$$

(ii) For $L \in \operatorname{sim}_{s}$, the module $\theta^{\text {out }} L$ has simple and finite type socle.

(iii) For $L \in \operatorname{sim}_{s}$ and $S=t^{-1}(L)=\operatorname{soc}\left(\theta^{\text {out }} L\right)$, we have $\left[\theta^{\text {out }} L: S\right]=n$.

Remark 27 Theorem 26 remains true if $\chi_{r}$ is also singular with $W_{\lambda} \subset W_{\mu}$ if we set $n=$ $\left|W_{\mu}\right| /\left|W_{\lambda}\right|$.

We keep the notation and assumptions of Theorem 26 for the rest of the section and start the proof with the following lemma. We freely use Lemma 12(ii), namely biadjunction between $\theta^{\text {on }}$ and $\theta^{\text {out }}$ and $\theta^{\text {on }} \theta^{\text {out }}=\mathrm{Id}^{\oplus n}$, freely.

Lemma 28 For a simple module $L$ with central character $\chi_{s}$ and a non-zero submodule or quotient $N$ of $\theta^{\text {out }} L$, we have $\theta^{\text {on }} N \cong L^{\oplus k}$ with $1 \leq k \leq n$. Moreover, for any simple module $S$, we have

$$
\operatorname{Hom}_{\mathfrak{g}}\left(S, \theta^{\text {out }} L\right) \cong \operatorname{Hom}_{\mathfrak{g}}\left(\theta^{\text {out }} L, S\right) .
$$

Proof We consider a quotient $N$, the proof for submodules being identical. By exactness of $\theta^{\text {on }}$, we find that $\theta^{\text {on }} N$ is a quotient of $L^{\oplus n}$. Since we have a non-zero morphism $\theta^{\text {out }} L \rightarrow N$, we have a non-zero morphism $L \rightarrow \theta^{\text {on }} N$, so we find $\theta^{\text {on }} N \neq 0$.

By the above, both sides of the proposed isomorphism are zero unless $\theta^{\text {on }} S$ is semisimple. In the latter case, we have

$$
\operatorname{Hom}_{\mathfrak{g}}\left(S, \theta^{\text {out }} L\right) \cong \operatorname{Hom}_{\mathfrak{g}}\left(\theta^{\text {on }} S, L\right) \cong \operatorname{Hom}_{\mathfrak{g}}\left(L, \theta^{\text {on }} S\right) \cong \operatorname{Hom}_{\mathfrak{g}}\left(\theta^{\text {out }} L, S\right),
$$

which concludes the proof.

Lemma 29 Let $M \in U-\bmod _{\chi_{s}}^{0}$ and $\operatorname{End}\left(\theta^{\text {out }}\right)$ be the set of natural endotransformations as in Lemma 13. Then evaluation yields a monomorphism

$$
\operatorname{End}\left(\theta^{\text {out }}\right) \hookrightarrow \operatorname{End}_{\mathfrak{g}}\left(\theta^{\text {out }} M\right), \quad \alpha \mapsto \alpha_{M}
$$

If $M$ is simple, then this monomorphism is an isomorphism. 
Proof Take a natural transformation $\alpha: \theta^{\text {out }} \Rightarrow \theta^{\text {out }}$. Then $\theta^{\text {on }}(\alpha)$ is a natural transformation $\mathrm{Id}^{\oplus n} \Rightarrow \mathrm{Id}^{\oplus n}$. By Lemma 13, we have End(Id $\left.{ }^{\oplus n}\right)=$ Mat $_{n}$ (End(Id)), with End(Id) consisting only of scalar multiples of the identity natural transformation Id $\Rightarrow$ Id. Hence a non-zero element in $\operatorname{End}\left(\mathrm{Id}^{\oplus n}\right)$ evaluated at a non-zero module always yields a non-zero morphism. We claim that $\theta^{o n}(\alpha)$ is not zero. It then follows that $\theta^{o n}(\alpha)$ evaluated at any module $M$ in $U-\bmod _{\chi_{s}}^{0}$ is not zero, so in particular $\alpha_{M}$ is not zero.

To conclude the proof of injectivity, we can therefore just observe that we have $\theta^{\text {on }}\left(\alpha_{\Delta_{\mu}}\right) \neq$ 0 . By Lemma 13, $\alpha_{\Delta_{\mu}}: P_{\lambda^{\prime}} \rightarrow P_{\lambda^{\prime}}$ is not zero and $n=\left[P_{\lambda^{\prime}}: L_{\lambda^{\prime}}\right]$. It follows from [27, 4.12(3)] that $\theta^{\text {on }}(\beta) \neq 0$, for any non-zero endomorphism $\beta$ of $P_{\lambda^{\prime}}$.

Now, let $M=L$ be simple. In this case

$$
\operatorname{dim} \operatorname{End}_{\mathfrak{g}}\left(\theta^{\text {out }} L\right)=\operatorname{dim} \operatorname{Hom}_{\mathfrak{g}}\left(L, L^{\oplus n}\right)=n .
$$

Hence, by Lemma 13 the dimensions of $\operatorname{End}_{\mathfrak{g}}\left(\theta^{\text {out }} L\right)$ and $\operatorname{End}\left(\theta^{\text {out }}\right)$ agree and the monomorphism must be an isomorphism.

Alternative proof We view $\theta^{\text {out }}$ as a functor from $U-\bmod _{\chi_{s}}^{0}$ to the full subcategory $\mathcal{C}$ of $U$ $\bmod _{\chi_{r}}$ of modules isomorphic to modules of the form $\theta^{\text {out }} N$, with $N$ in $U-\bmod _{\chi_{s}}^{0}$. By construction, for this interpretation of $\theta^{\text {out }}$, $\operatorname{End}\left(\theta^{\text {out }}\right)$ coincides with the algebra of natural transformations as in Lemma 13. Now the restriction of $\theta^{\text {on }}$ to $\mathcal{C}$ has image contained in $U$ - $\bmod _{\chi_{s}}^{0}$. Moreover, it also follows that $\left(\theta^{\text {on }}, \theta^{\text {out }}\right)$ is still a pair of bi-adjoint functors, in the above interpretation.

Now adjunction and evaluation yields a commutative diagram

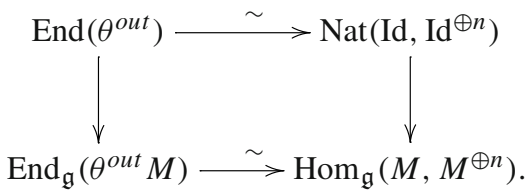

By construction, we have to interpret Nat $\left(\mathrm{Id}, \mathrm{Id}^{\oplus n}\right)$ as natural transformations with Id viewed as an endofunctor of $U-\bmod _{\chi_{s}}^{0}$. However, this does not differ from the space of natural transformations as in Lemma 13. Hence, $\operatorname{dim} \operatorname{Nat}\left(\operatorname{Id}, \operatorname{Id}^{\oplus n}\right)=n$ and the space just consists of linear combinations of identity natural transformations of Id. In particular, the right vertical arrow is a monomorphism. This forces the left vertical arrow to be a monomorphism as well.

If $M$ is simple, the right vertical arrow is, clearly, an isomorphism.

Lemma 30 Fix a simple module $L$ with central character $\chi_{s}$. Then $\theta^{\text {out }} L$ has simple top and socle $S$. Moreover, we have $\left[\theta^{\text {out }} L: S\right]=n$ and $\theta^{\text {on }} S=L$.

Proof By combining [41, Theorem 12] and Lemma 13, it follows that the algebra End( $\left.\theta^{\text {out }}\right)$ is isomorphic to the coinvariant algebra of $W_{\mu}$, where $\mu \in \eta^{-1}\left(\chi_{s}\right)$ is dominant. In particular, it has simple socle. By Lemma 28, the top and socle of $\theta^{\text {out }} S$ are isomorphic. If the top and socle are not simple then this clearly contradicts the fact that $\operatorname{End}\left(\theta^{\text {out }}\right) \cong \operatorname{End}_{\mathfrak{g}}\left(\theta^{\text {out }} L\right)$ (see Lemma 29) must have simple socle.

Lemma 7 implies that

$$
n=\operatorname{dim} \operatorname{Hom}_{\mathfrak{g}}\left(L, L^{\oplus n}\right)=\operatorname{dim}_{\operatorname{End}_{\mathfrak{g}}}\left(\theta^{\text {out }} L\right) \leq\left[\theta^{\text {out }} L: S\right] .
$$

On the other hand, we have $\theta^{\text {on }} S=L^{\oplus k}$, for some $k \geq 1$, by Lemma 28. Applying the exact functor $\theta^{\text {on }}$ on $\theta^{\text {out }} L$ thus yields

$$
n=\left[L^{\oplus n}: L\right] \geq k\left[\theta^{\text {out }} L: S\right] .
$$


Hence we find $k=1$ and $\left[\theta^{\text {out }} L: S\right]=n$.

Proof of Theorem 26 Take some simple module $S \in \operatorname{sim}_{r}^{s}$, then $\theta^{\text {on }} S$ is non-zero and has finite type radical. In particular, there exists a simple module $L$ with non-zero morphisms $\theta^{\text {on }} S \rightarrow L$ and $S \rightarrow \theta^{\text {out }} L$. By Lemma 30, we have $t(S)=L$. Also by Lemma 30, the map $t$ is a bijection and parts (i) and (iii) follow.

Now we prove part (ii). By Theorem 16 it is sufficient to prove that every submodule of $\theta^{\text {out }} L$ contains a simple subquotient of Gelfand-Kirillov dimension $\operatorname{GK}(L)$. For a submodule $N \subset \theta^{\text {out }} L$, Lemma 28 implies there exists a non-zero morphism $L \hookrightarrow \theta^{\text {on }} N$. By adjunction, there is a non-zero morphism $\theta^{\text {out }} L \rightarrow N$. Since $\theta^{\text {out }} L$ has finite type radical and all simple quotients have Gelfand-Kirillov dimension GK $(L)$ by Lemma 17, we find that $N$ contains a simple subquotient of Gelfand-Kirillov dimension $\operatorname{GK}(L)$.

For a simple $U$-module $S$, we denote by $S \otimes \mathcal{F}$ the full subcategory of the category of all $\mathfrak{g}$-modules consisting of all modules isomorphic to the ones of the form $S \otimes E$, with $E \in \mathcal{F}$.

Proposition 31 If, for every simple module $S$ with regular central character, all modules in $S \otimes \mathcal{F}$ have finite type socle (resp. a GK-complete filtration), then, for every simple module $L$, all modules in $L \otimes \mathcal{F}$ have finite type socle (resp. a GK-complete filtration).

Proof By Theorem 26, for every simple module $L$, there exists a simple module $S$ with regular central character and $V \in \mathcal{F}$ such that $L$ is a direct summand of $S \otimes V$.

Hence each module in $L \otimes \mathcal{F}$ is a direct summand of a module in $S \otimes \mathcal{F}$. The conclusion for socles follows from observing that any submodule of a module with finite type socle has finite type socle. The conclusion about GK-complete filtrations follows from Lemmata 17 and 10.

Corollary 32 Let $w_{0}^{\prime}$ be the longest element of a Coxeter subgroup $W^{\prime} \subset W$ and $S$ a simple module with integral regular central character. If $\theta_{w_{0}^{\prime}} S \neq 0$, then $\theta_{x} S$ has finite type socle, for all $x \in W^{\prime}$.

Proof We fix some dominant and integral $\mu$ such that $W_{\mu}=W^{\prime}$. Then we can write $\theta_{w_{0}^{\prime}}=$ $\theta^{\text {out }} \theta^{\text {on }}$ using the notation as in Theorem 26. From Theorem 26 it follows that $\theta_{w_{0}^{\prime}} S$ has finite type socle and $S=\operatorname{soc}\left(\theta_{w_{0}^{\prime}} S\right)$. From Kazhdan-Lusztig combinatorics it follows that $\theta_{x} \theta_{w_{0}^{\prime}}$ is a direct sum of copies of $\theta_{w_{0}^{\prime}}$. Hence $\theta_{x} S$ is a submodule of a direct sum of copies of $\theta_{w_{0}^{\prime}} S$ and also has finite type socle.

\section{Direct sums of copies of $\mathfrak{s l}_{2}$}

In this section, we fix $j \in \mathbb{Z}_{>0}$ and set $\mathfrak{g}=\mathfrak{s l}_{2}(\mathbb{C})^{\oplus j}$. For this $\mathfrak{g}$, we can strengthen Theorem 23 as follows.

Theorem 33 For $\mathfrak{g}=\mathfrak{s l}_{2}(\mathbb{C})^{\oplus j}$ and any simple $\mathfrak{g}$-module $S$ and $E \in \mathcal{F}$, the module $S \otimes E$ has a $G K$-complete filtration. Moreover, if $j=1$, then $S \otimes E$ even has finite length.

We label the $j$ copies of $\mathfrak{s l}_{2}$ by the index $1 \leq i \leq j$. We denote by $\epsilon_{i} \in \mathfrak{h}^{*}$ the weight half the positive root of the $i$ th copy. Accordingly, we write $\lambda=\sum \lambda_{i} \epsilon_{i}$, with $\lambda_{i} \in \mathbb{C}$, for $\lambda \in \mathfrak{h}^{*}$. The Weyl group acts on $\mathfrak{h}^{*}$ by changing signs for the $\lambda_{i}$. For $1 \leq i \leq j$, we denote by $V_{i} \cong \mathbb{C}^{2}$ the natural module for the $i$ th copy of $\mathfrak{s l}_{2}$, interpreted as a module for $\mathfrak{g}$ in the obvious way. So, in this way we have $\operatorname{supp}\left(V_{i}\right)=\left\{\epsilon_{i},-\epsilon_{i}\right\}$. 
Fix $i$ such that $1 \leq i \leq j$. Denote by $\bmod _{i}$ the full subcategory of $U$-mod consisting of all modules $M$ on which the $i$ th copy of $\mathfrak{s l}_{2} \subset \mathfrak{g}$ acts locally finitely. Clearly, $-\otimes V_{i}$ restricts to an endofunctor on $\bmod _{i}$. We also denote by $\operatorname{sim}^{i}$ the set of isomorphism classes of simple $\mathfrak{g}$-modules which are not in $\bmod _{i}$.

Lemma 34 Take a simple module $S \in \operatorname{sim}^{i}$. Then we have $S_{1}, S_{2} \in \operatorname{sim}^{i}$ and a filtration of $T=S \otimes V_{i}$

$$
0 \subset F_{2} T \subset F_{1} T \subset T, \text { with } F_{2} T \cong S_{2} \text { and } T / F_{1} T \cong S_{1} .
$$

If $j=1$, so $\mathfrak{g}=\mathfrak{s l}_{2}$, the module $S \otimes V_{i}$ has finite length.

Proof Let $\chi$ be the central character of $S$. We take a dominant $\lambda \in \eta^{-1}(\chi)$, which means $\lambda_{i} \notin \mathbb{Z}_{<0}$.

Assume first that $\lambda_{i} \notin \mathbb{Z}$. It follows from

$$
\Delta_{\lambda} \otimes V_{i} \cong \Delta_{\lambda+\epsilon_{i}} \oplus \Delta_{\lambda-\epsilon_{i}}
$$

and Lemmata 11 and 12(i) that $-\otimes V_{i}$ restricted to $U-\bmod _{\chi}$ decomposes into a direct sum of an equivalence with $U-\bmod _{\eta\left(\lambda+\epsilon_{i}\right)}$ and $U-\bmod _{\eta\left(\lambda-\epsilon_{i}\right)}$. Hence $S \otimes V_{i}$ is a direct sum of two simple modules. That both are in $\operatorname{sim}^{i}$ follows by adjunction.

If $\lambda_{i} \in \mathbb{Z}_{>1}$, the argument of the previous paragraph still applies.

If $\lambda=1$, we still have Eq. (5.1). We write $\theta^{o n}=F_{\lambda}^{\lambda-\epsilon_{i}}$, as in Sect. 4.3. By Lemmata 11 and 12(i), $S \otimes V_{i}$ is a direct sum of a simple module and $\theta^{o n} S$. By Theorem 26(i), the latter module is zero or simple.

Finally, we consider $\lambda_{i}=0$, which implies

$$
\Delta_{\lambda} \otimes V_{i} \cong P_{\lambda-\epsilon_{i}} .
$$

We write $\theta^{\text {out }}=F_{\lambda}^{\lambda-\epsilon_{i}}$ as in Sect. 4.3. Lemma 11 implies that $S \otimes V_{i} \cong \theta^{\text {out }} S$. That $S \otimes V_{i}$ is of the desired form then follows from Theorem 26(i).

Since $\theta^{\text {on }} \theta^{\text {out }}=\mathrm{Id}^{\oplus 2}$, it follows that $\theta^{\text {on }} F_{1} T / F_{2} T=0$.

For $\mathfrak{g}=\mathfrak{s l}_{2}$, Eq. (2.2) thus implies that $F_{1} T / F_{2} T$ must be a direct sum of a number of copies of one fixed simple finite dimensional module. Since $T$ is noetherian, it follows that $F_{1} T / F_{2} T$ is finitely generated and thus finite dimensional.

Lemma 35 Let $S$ be a simple $U$-module in $\bmod _{i}$. Then $S \otimes V_{i}$ is a direct sum of simple modules in $\bmod _{i}$.

Proof Let $\chi$ be the central character of $S$. The explicit description of the generator of the center in the universal enveloping algebra of $\mathfrak{s l}_{2}$ implies that any dominant $\lambda \in \eta^{-1}(\chi)$ satisfies $\lambda_{i} \in \mathbb{Z}_{>0}$. It then follows from the same arguments as in Lemma 34 that $S \otimes V_{i}$ is simple, if $\lambda_{i}=1$, and is a direct sum of two simple modules if $\lambda_{i}>1$.

Corollary 36 For any simple $\mathfrak{g}$-module $S$ and $1 \leq i \leq j$, the module $S \otimes V_{i}$ has a GK-complete filtration.

Proof By Lemma 35, we can assume $S \in \operatorname{sim}^{i}$.

We fix an arbitrary $d \in \mathbb{N}$. First we define an equivalence relation on simple $\mathfrak{g}$-modules in $\operatorname{sim}^{i}$ with Gelfand-Kirillov dimension $d$. We let $\sim$ be the minimal equivalence relation generated by relation $S \sim S^{\prime}$ if we have a non-zero morphism $S \otimes V_{i} \rightarrow S^{\prime}$ or $S^{\prime} \rightarrow S \otimes V_{i}$. Since $V_{i}$ is self-dual, this is indeed an equivalence relation. 
Take a simple module $S$ with $\mathrm{GK}(S)=d$ and e $(S)$ minimal in its equivalence class. Set $e:=\mathrm{e}(S)$. By Eq. (2.3), we have e $\left(S \otimes V_{i}\right)=2 e$. The simple modules $S_{1}, S_{2}$ in Lemma 34 thus satisfy e $\left(S_{1}\right)+\mathrm{e}\left(S_{2}\right) \leq 2 e$ by Lemma 8(ii). By minimality of $e$, we thus have $\mathrm{e}\left(S_{1}\right)=e=\mathrm{e}\left(S_{2}\right)$. With notation of Lemma 34, it follows that we have $\mathrm{GK}\left(F_{1} T / F_{2} T\right)<d$. Consequently $S \otimes V_{i}$ has a GK-complete filtration.

The above paragraph also shows that $S_{1}$ and $S_{2}$ are the unique simple modules which appear as submodules or quotients of $S \otimes V_{i}$, by Lemma 17. Applying the same procedure to $S_{1}, S_{2}$ iteratively shows that every simple module in the equivalence class of $S$ has Bernstein number e $(S)$. Hence the condition for $S$ to have minimal e $(S)$ in its equivalence class was not actually a restriction. This completes the proof.

Proof of Theorem 33 Every finite dimensional $\mathfrak{g}$-module is a direct sum of a module of the form

$$
\bigotimes_{1 \leq i \leq j} V_{i}^{\otimes \alpha_{i}}
$$

for suitable $\alpha_{i} \in \mathbb{N}$. The statement about GK-complete filtrations thus follows from Corollary 36. The statement for socles then follows from Corollary 20.

The claim for $\mathfrak{s l}_{2}$ follows from Lemma 34 .

\section{Lie superalgebra preliminaries}

\subsection{Setup}

In this section, we will introduce the setup of Lie superalgebras. We refer to [12,40] for more details. From now on we work over the field $\mathbb{C}$ of complex numbers.

We let $\tilde{\mathfrak{g}}$ be a finite-dimensional Lie superalgebra with $\mathbb{Z}_{2}$-graded decomposition

$$
\widetilde{\mathfrak{g}}=\widetilde{\mathfrak{g}}_{\overline{0}} \oplus \widetilde{\mathfrak{g}}_{\overline{1}} .
$$

From now on we assume that $\mathfrak{g}:=\tilde{\mathfrak{g}}_{\overline{0}}$ is a reductive Lie algebra of type $A$ and that $\tilde{\mathfrak{g}}_{\overline{1}}$ is a semi-simple $\tilde{\mathfrak{g}}_{0}$-module. The Weyl group $W$ of $\tilde{\mathfrak{g}}$ is defined to be the Weyl group of the reductive Lie algebra $\mathfrak{g}$ and we keep notation and terminology of Sect. 2.4.

Let $V=V_{\overline{0}} \oplus V_{\overline{1}}$ be a superspace. For a given homogeneous element $v \in V_{i}$, where $i \in \mathbb{Z}_{2}$, we let $\bar{v}=i$ denote its parity. We denote the parity change functor by $\Pi$ on the category of superspaces, cf. [12, Section 1.1.1]. For a $\mathbb{Z}_{2}$-graded associative algebra $A$, we denote by $A$-smod the category of all finitely generated $\mathbb{Z}_{2}$-graded modules with grading preserving homomorphisms. Note that when $A$ is reduced, i.e. $A=A_{\overline{0}}$, we have

$$
A \text {-smod } \cong A-\bmod \oplus \Pi(A-\bmod )
$$

\subsection{Categories of (super)modules}

We denote the universal enveloping algebras of our Lie (super)algebras by $\widetilde{U}:=U(\tilde{\mathfrak{g}})$ and $U:=U(\mathfrak{g})=U\left(\widetilde{\mathfrak{g}}_{\overline{0}}\right)$. Let $Z(\widetilde{\mathfrak{g}})$ and $Z(\mathfrak{g})$ denote the center of $\widetilde{U}$ and $U$, respectively. Also, the center of $\mathfrak{g}$ is denoted by $\mathfrak{z}(\mathfrak{g})$.

We set $\tilde{\mathfrak{g}}$-smod $=\widetilde{U}$-smod and $\mathfrak{g}$-smod $=U$-smod. By our assumptions in Sect. 6.1 a supermodule $M$ over a Lie superalgebra is not necessary isomorphic to its parity changed 
counterpart П $M$. We have the exact restriction, induction and coinduction functors

$$
\begin{aligned}
& \operatorname{Res}:=\operatorname{Res} \frac{\tilde{\mathfrak{g}}}{\tilde{\mathfrak{g}}_{0}}: \widetilde{\mathfrak{g}}_{\text {-smod }} \rightarrow \widetilde{\mathfrak{g}}_{0} \text {-smod, } \\
& \text { Ind }:=\operatorname{Ind} \tilde{\mathfrak{g}}_{\overline{0}} \tilde{\mathfrak{g}}_{\overline{0}}: \widetilde{\mathfrak{g}}_{0} \text {-smod } \rightarrow \widetilde{\mathfrak{g}} \text {-smod, } \\
& \text { Coind }:=\operatorname{Coind} \widetilde{\mathfrak{g}}_{\overline{0}}^{\widetilde{\mathfrak{g}}}: \widetilde{\mathfrak{g}}_{\overline{0}} \text {-smod } \rightarrow \widetilde{\mathfrak{g}} \text {-smod. }
\end{aligned}
$$

By [2, Theorem 2.2] (also see [21]), the functors Ind and Coind are isomorphic, up to the equivalence given by tensoring with the one-dimensional $\mathfrak{g}$-module on the top degree subspace of $\Lambda \widetilde{\mathfrak{g}}_{\overline{1}}$.

By abusing notation we let $\mathcal{O}$ be the full subcategory of $U$-smod of weight modules which are locally $\mathfrak{b}$-finite. In principle, we would have to write $\mathcal{O} \oplus \Pi \mathcal{O}$ for this category, in order to be consistent with Sect. 2.4. Similarly, we let $L_{\lambda}$ denote the simple $\mathfrak{g}$-module in unspecified parity. We denote by $\widetilde{\mathcal{O}}$ the full subcategory of $\widetilde{U}$-smod of supermodules which restrict to $\mathcal{O}$.

For a given $\widetilde{\mathfrak{g}}$-supermodule (resp. $\mathfrak{g}$-supermodule) $X$, we denote its $\widetilde{U}$-annihilator (resp. $U$-annihilator) by $\operatorname{Ann}_{\widetilde{U}}(X)$ (resp. $\left.\operatorname{Ann}_{U}(X)\right)$. The following theorem is due to Duflo in [17].

Theorem 37 Let $V$ be a simple $\mathfrak{g}$-supermodule. Then there exist $\lambda \in \mathfrak{h}^{*}$ such that $\operatorname{Ann}_{U}(V)=$ $A n n_{U}\left(L_{\lambda}\right)$.

We let $\mathcal{F}$ denote the category of finite dimensional semisimple $\mathfrak{g}$-supermodules, and we let $\widetilde{\mathcal{F}}$ denote the category of finite-dimensional $\widetilde{\mathfrak{g}}$-supermodules which restrict to objects in $\mathcal{F}$. Then $\mathcal{F}$ and $\widetilde{\mathcal{F}}$ are exactly the categories of finite-dimensional weight $\mathfrak{g}$-supermodules and $\widetilde{\mathfrak{g}}$-supermodules, respectively.

For a $\tilde{\mathfrak{g}}$-supermodule $M$, we denote by

- $\tilde{\mathcal{F}} \otimes M$ the category of $\widetilde{\mathfrak{g}}$-supermodules of the form $V \otimes M$, with $V \in \widetilde{\mathcal{F}}$;

- $\operatorname{add}(\tilde{\mathcal{F}} \otimes M)$ the category of all supermodules isomorphic to direct summands of objects in $\widetilde{\mathcal{F}} \otimes M$;

- $\langle\widetilde{\mathcal{F}} \otimes M\rangle$ the abelian category of all supermodules isomorphic to subquotients of supermodules in $\widetilde{\mathcal{F}} \otimes M$.

We let $\operatorname{Coker}(\tilde{\mathcal{F}} \otimes M)$ denote the coker-category of $M$, that is the full subcategory of the category of all $\mathfrak{g}$-supermodules, which consists of all modules $N$ that have a presentation

$$
X \rightarrow Y \rightarrow N \rightarrow 0,
$$

where $X, Y \in \operatorname{add}(\widetilde{\mathcal{F}} \otimes M)$. Similarly we define analogous full subcategories of $\mathfrak{g}$ supermodules, cf [38].

\subsection{Harish-Chandra bimodules}

Following [9, § 3.2], we will consider a type of Harish-Chandra bimodules where the left action is by a Lie superalgebra and the right action by the underlying Lie algebra. The corresponding category will be an essential tool in our study of the rough structure.

We write $\widetilde{U}$-smod- $U$ for $\widetilde{U} \otimes U^{\text {op }}$-smod. For a bimodule $Z$ in this category, the $\mathfrak{g}$-module $Z^{\text {ad }}$ is the restriction of $Z$ to the adjoint action of $\mathfrak{g}$. This is the restriction via

$$
U \hookrightarrow \widetilde{U} \otimes U^{\text {op }}, \quad X \mapsto X \otimes 1-1 \otimes X, \text { for all } X \in \mathfrak{g} .
$$

Let $\mathcal{B}$ denote the full subcategory of $\widetilde{U}$-smod- $U$ of bimodules $N$ for which $N^{\text {ad }}$ is a direct sum of modules in $\mathcal{F}$ with finite multiplicities. For a two-sided ideal $J \subset U$, we let $\mathcal{B}(J)$ 
denote the full subcategory of $\mathcal{B}$ of bimodules $X$ such that $X J=0$. We have the functor

$$
\mathcal{L}(-,-):(U \text {-smod })^{\text {op }} \times \widetilde{U} \text {-smod } \rightarrow \mathcal{B},
$$

where $\mathcal{L}(M, N)$ is the maximal submodule of $\operatorname{Hom}_{\mathbb{C}}(M, N)$ which belongs to $\mathcal{B}$.

By slight abuse of notation, we will use the same notation $\mathcal{L}$ for the corresponding functor applied to the case $\tilde{\mathfrak{g}}=\mathfrak{g}$. Let $M$ be a $U$-module, then the $\mathfrak{g}$-action on $M$ defines a bimodule homomorphism from $U$ to $\mathcal{L}(M, M)$ with kernel $\operatorname{Ann}_{U}(M)$. Hence we have a canonical monomorphism

$$
U / \operatorname{Ann}_{U}(M) \hookrightarrow \mathcal{L}(M, M) .
$$

One says that Kostant's problem for $M$ has a positive solution if (6.1) is an isomorphism, see $[22,28,37]$. Then we have the following variation of [39, Theorem 3.1] established in [9, Theorem 3.1].

Theorem 38 Consider $M \in U$-mod with central character and set $I:=\operatorname{Ann}_{U}(M)$. If the Kostant problem for $M$ has a positive solution and $M$ is projective in $\langle\mathcal{F} \otimes M\rangle$, then

$$
-\otimes_{U} M: \quad \mathcal{B}(I) \rightarrow \operatorname{Coker}(\tilde{\mathcal{F}} \otimes \operatorname{Ind}(M))
$$

is an equivalence of categories with inverse $\mathcal{L}(M,-)$.

For $E \in \widetilde{\mathcal{F}}$, we recall that $E \otimes \widetilde{U}$ is equipped with a natural $\tilde{\mathfrak{g}}$-bimodule structure as in [3, Section 2.2] and [15, Section 2.4]:

$$
X(v \otimes u) Y=(X v) \otimes(u Y)+(-1)^{\bar{X} \cdot \bar{v}} v \otimes(X u Y),
$$

for all homogeneous $X, Y \in \tilde{\mathfrak{g}}, v \in E$ and $u \in \tilde{U}$.

\section{Rough structure of simple $\tilde{\mathfrak{g}}$-supermodules}

\subsection{Motivation}

Two fundamental problems in representation theory of a group or a ring are:

- classification of simple modules;

- understanding how all modules are constructed from simple modules.

A natural subproblem of the second problem is determination of multiplicities of simple subquotients in a given module. In the general case, certain multiplicities might be infinite or depend on the choice of filtration.

The paper [30] studied the structure of a certain class of modules over Lie algebras, called generalized Verma modules. These modules are obtained by parabolic induction from simple modules over "smaller" Lie algebras. It turned out that, given a generalized Verma module, there is a natural class of simple modules (defined using a certain comparability of annihilators) whose multiplicities in the generalized Verma module are well-defined, finite and computable using Kazhdan-Lusztig combinatorics. This was called the rough structure of generalized Verma modules. The most general, to date, result on the rough structure of generalized Verma modules was obtained in [38, Section 11.8].

In the study of Lie superalgebras, it is natural to ask about the rough structure of simple supermodule as modules over the even part of the Lie superalgebras. In [10], for classical Lie superalgebras of type $I$, it was shown that 
- the classification of simple supermodules can be reduced to the classification of simple modules over the even part of the superalgebra;

- the rough structure of a simple supermodule as a module over the even part of the Lie superalgebra can be described in terms of combinatorics of category $\mathcal{O}$.

The goal of this section is to address these problems for Lie superalgebra not necessarily of type I, but with underlying Lie algebra of type $A$.

\subsection{Coker categories for induced modules}

With Theorem 38 as the main tool, this subsection shall proceed with the study of cokercategories of induced modules along the lines of [38, Section 11.6].

For simplicity, we will work with regular integral central characters by following [38, Remark 76]. The general case then follows by standard techniques, in particular using translations out and onto the walls and the equivalences from [14].

We start with reviewing and adapting the setup of [38, Section 11.6] to the present paper. Note that we work in the generality when $\mathfrak{g}$ is reductive. Each central element of $\mathfrak{g}$ acts as a scalar on any simple $\mathfrak{g}$-module, therefore we will work only with $\mathfrak{g}$-modules on which the action of the center of $\mathfrak{g}$ is semi-simple. We assume that the center of $\mathfrak{g}$ belongs to any Cartan subalgebra and we call a weight integral if it appears in some simple finite dimensional $\mathfrak{g}$-module.

Let $L$ be a simple $\mathfrak{g}$-supermodule which admits a regular and integral central character. By Theorem 37, there is a dominant weight $v$ and an element $\sigma \in W$ such that $\operatorname{Ann}_{U}(L)=$ $\operatorname{Ann}_{U}\left(L_{\sigma \nu}\right)$. We may assume that $\sigma$ is contained in a right cell associated with a parabolic subalgebra $\mathfrak{p} \subseteq \mathfrak{g}$ as in [38, Remark 14]. Therefore there is a dominant weight $\mu$ such that the parabolic block $\mathcal{O}_{\mu}^{\mathfrak{p}}$ contains exactly one simple module $L_{y \mu}$ and this module is projective, see e.g. [26, 3.1]. As $y \mu=y x \mu$, for any $x$ in the stabilizer of $\mu$, without loss of generality, we may assume that $y s<y$, for all simple reflection $s$ with $s \mu=\mu$. With this assumption, we have that $L_{y \mu}$ is the translation of $L_{y 0}$ to the $\mu$-wall.

Tensoring, if necessary, with finite dimensional modules, without loss of generality we may assume that $\mu$ is generic in the sense of [37, Subsection 5.3]. Let $F$ be a projective functor given in [38, Proposition 62]. A simple top of $F L$ is defined uniquely, up to equivalence given by the action of projective functors. We fix one representative $\bar{N}$ in such an equivalence class. We refer the reader to [38, Section 11] for more details of our setup. In particular, we have that

$$
I:=\operatorname{Ann}_{U}(\bar{N})=\operatorname{Ann}_{U}\left(L_{y \mu}\right) .
$$

The following theorem, which is [38, Theorem 66], is our main tool to study the rough structure for Lie algebras of type $A$.

Theorem 39 Let $\mathcal{F}$ denote the category of finite-dimensional weight $\mathfrak{g}$-supermodules. Then the functor

$$
\Xi:=\mathcal{L}(\bar{N},-) \otimes_{U} L_{y \mu}: \operatorname{Coker}(\mathcal{F} \otimes \bar{N}) \rightarrow \operatorname{Coker}\left(\mathcal{F} \otimes L_{y \mu}\right)
$$

is an equivalence of categories.

By [38, Lemma 67], categories $\operatorname{Coker}(\mathcal{F} \otimes \bar{N})$ and $\operatorname{Coker}\left(\mathcal{F} \otimes L_{y \mu}\right)$ are both admissible in the sense of [38, Section 6.3].

The following lemma combines [38, Lemma 63] and [38, Proposition 65(ii)]. 
Lemma 40 The objects $L_{y \mu}$ and $\bar{N}$ are projective in $\left\langle\mathcal{F} \otimes L_{y \mu}\right\rangle$ and $\langle\mathcal{F} \otimes \bar{N}\rangle$, respectively.

Now we can formulate the following equivalence of coker-categories.

Corollary 41 The functor

$$
\widetilde{\Xi}:=\mathcal{L}(\bar{N},-) \otimes_{U} L_{y \mu}: \operatorname{Coker}(\widetilde{\mathcal{F}} \otimes \operatorname{Ind}(\bar{N})) \rightarrow \operatorname{Coker}\left(\widetilde{\mathcal{F}} \otimes \operatorname{Ind}\left(L_{y \mu}\right)\right)
$$

is an equivalence sending $\operatorname{Ind}(\bar{N})$ to $\operatorname{Ind}\left(L_{y \mu}\right)$.

Proof With $I$ from 7.1, we claim that

$$
\operatorname{Coker}(\widetilde{\mathcal{F}} \otimes \operatorname{Ind}(\bar{N})) \stackrel{\mathcal{L}(\bar{N},-)}{\longrightarrow} \mathcal{B}(I) \stackrel{-\otimes_{U} L_{y \mu}}{\longrightarrow} \operatorname{Coker}\left(\tilde{\mathcal{F}} \otimes \operatorname{Ind}\left(L_{y \mu}\right)\right)
$$

are equivalences. Indeed, recall that Kostant's problem has a positive solution for $L_{y \mu}$ and $\bar{N}$ by [38, Theorem 60(iii)] and [38, Proposition 65(iii)], respectively. Now Lemma 40 and Theorem 38 imply the claim.

Finally, since Kostant's problem has a positive solution for $\bar{N}$, it follows that

$$
\mathcal{L}(\bar{N}, \operatorname{Ind}(\bar{N})) \otimes_{U} L_{y \mu} \cong \widetilde{U} \otimes_{U} \mathcal{L}(\bar{N}, \bar{N}) \otimes_{U} L_{y \mu} \cong \operatorname{Ind}\left(L_{y \mu}\right),
$$

as $\widetilde{U}$-modules. This completes the proof.

\subsection{Rough structure of induced $\mathfrak{g}$-supermodules}

In this subsection, we obtain a description of the rough structure of induced supermodules.

Lemma 42 The equivalence $\widetilde{\Xi}$ in Corollary 41 gives rise to a bijection between the sets of isomorphism classes of objects in the categories $\operatorname{add}(\widetilde{\mathcal{F}} \otimes \operatorname{Ind}(\bar{N}))$ and $\operatorname{add}\left(\widetilde{\mathcal{F}} \otimes \operatorname{Ind}\left(L_{y \mu}\right)\right)$. These two categories are, respectively, the categories of projective objects in $\operatorname{Coker}(\widetilde{\mathcal{F}} \otimes$ $\operatorname{Ind}(\bar{N}))$ and $\operatorname{Coker}\left(\widetilde{\mathcal{F}} \otimes \operatorname{Ind}\left(L_{y \mu}\right)\right)$.

Proof For a given $E \in \widetilde{\mathcal{F}}$, we have

$$
\begin{aligned}
\widetilde{\Xi}(E \otimes \operatorname{Ind}(\bar{N})) & \cong \mathcal{L}(\bar{N}, \operatorname{Ind}(\operatorname{Res}(E) \otimes \bar{N})) \otimes_{U} L_{y \mu} \\
& \cong \widetilde{U} \otimes_{U}\left(\operatorname{Res}(E) \otimes U \otimes_{U} U / I\right) \otimes_{U} L_{y \mu} \\
& \cong \operatorname{Ind}\left(\operatorname{Res}(E) \otimes L_{y \mu}\right) \\
& \cong E \otimes \operatorname{Ind}\left(L_{y \mu}\right) .
\end{aligned}
$$

Here the first and the last isomorphisms use [31, Proposition 6.5], which states that

$$
E \otimes \operatorname{Ind}(X) \cong \operatorname{Ind}(\operatorname{Res}(E) \otimes X),
$$

for any $\mathfrak{g}$-module $X$, the second isomorphism uses [27, Section 6.8] and the isomorphism $\mathcal{L}(\bar{N}, \bar{N}) \cong U / I$ and the third isomorphism uses (7.1).

That $\operatorname{add}(\widetilde{\mathcal{F}} \otimes \widetilde{U} / \widetilde{U} I)$ is the full subcategory of projective modules in $\mathcal{B}(I)$ is shown in [9, Proof of Theorem 3.1]. The claim follows.

As a consequence, see [38, Section 11], $\widetilde{\Xi}$ induces a bijection

$$
\widetilde{\Xi}: \operatorname{Irr}^{\widetilde{\mathfrak{g}}}(\operatorname{Coker}(\tilde{\mathcal{F}} \otimes \operatorname{Ind}(\bar{N}))) \rightarrow \operatorname{Irr}^{\tilde{\mathfrak{g}}}\left(\operatorname{Coker}\left(\widetilde{\mathcal{F}} \otimes \operatorname{Ind}\left(L_{y \mu}\right)\right)\right),
$$

between the sets of isomorphism classes of simple $\tilde{\mathfrak{g}}$-supermodule quotients of simple objects in $\operatorname{Coker}(\widetilde{\mathcal{F}} \otimes \operatorname{Ind}(\bar{N}))$ and $\operatorname{Coker}\left(\widetilde{\mathcal{F}} \otimes \operatorname{Ind}\left(L_{y \mu}\right)\right)$. 
For $S \in \operatorname{Irr}^{\tilde{\mathfrak{g}}}(\operatorname{Coker}(\tilde{\mathcal{F}} \otimes \operatorname{Ind}(\bar{N})))$, we define

$$
\widetilde{L}_{S}:=\widetilde{\Xi}(S) \in \widetilde{\mathcal{O}} .
$$

We are now in a position to state the first main result of this section which describes rough structure of induced modules in terms of category $\widetilde{\mathcal{O}}$ combinatorics.

Theorem 43 (Rough structure of induced modules) For any module $E \in \widetilde{\mathcal{F}}$ and any module $S \in \operatorname{Irr}^{\widetilde{\mathfrak{g}}}(\operatorname{Coker}(\widetilde{\mathcal{F}} \otimes \operatorname{Ind}(\bar{N})))$, we have

$$
[\operatorname{Ind}(\operatorname{Res}(E) \otimes \bar{N}): S]=\left[\operatorname{Ind}\left(\operatorname{Res}(E) \otimes L_{y \mu}\right): \widetilde{L}_{S}\right] .
$$

Proof The proof of Eq. (7.4) follows from a similar argument using $\Xi$ as given in [38, Theorem 72]. To see this, let $\widetilde{Q}_{S}$ and $\widetilde{Q}_{L}$ denote the indecomposable projective objects in the categories $\operatorname{Coker}(\widetilde{\mathcal{F}} \otimes \operatorname{Ind}(\bar{N}))$ and $\operatorname{Coker}\left(\widetilde{\mathcal{F}} \otimes \operatorname{Ind}\left(L_{y \mu}\right)\right)$ with tops $S$ and $\widetilde{L}_{S}$, respectively. By (7.3) we have

$$
\begin{aligned}
& {[\operatorname{Ind}(\operatorname{Res}(E) \otimes \bar{N}): S]=\operatorname{dim} \operatorname{Hom}_{U}\left(\widetilde{Q}_{S}, \operatorname{Ind}(\operatorname{Res}(E) \otimes \bar{N})\right)} \\
& =\operatorname{dim} \operatorname{Hom}_{U}\left(\widetilde{Q}_{L}, \operatorname{Ind}\left(\operatorname{Res}(E) \otimes L_{y \mu}\right)\right) \\
& =\left[\operatorname{Ind}\left(\operatorname{Res}(E) \otimes L_{y \mu}\right): \widetilde{L}_{S}\right] \text {. }
\end{aligned}
$$

The claim follows.

\subsection{Rough structure of simple $\tilde{\mathfrak{g}}$-supermodules}

In this subsection, we give a description of the rough structure of restrictions to $\mathfrak{g}$ of simple $\widetilde{\mathfrak{g}}$-supermodules in $\operatorname{Irr}{ }^{\tilde{\mathfrak{g}}}(\operatorname{Coker}(\widetilde{\mathcal{F}} \otimes \operatorname{Ind}(\bar{N})))$.

Lemma 44 There is an isomorphism Res $\circ \widetilde{\Xi} \cong \Xi \circ$ Res of functors.

Proof This follows directly from the definitions.

We have a bijection

$$
\Xi: \operatorname{Irr}^{\mathfrak{g}}(\operatorname{Coker}(\mathcal{F} \otimes \bar{N})) \rightarrow \operatorname{Irr}^{\mathfrak{g}}\left(\operatorname{Coker}\left(\mathcal{F} \otimes L_{y \mu}\right)\right),
$$

induced by $\Xi$, between the sets of isomorphism classes of the simple $\mathfrak{g}$-quotients of simple objects in $\operatorname{Coker}(\mathcal{F} \otimes \bar{N})$ and in $\operatorname{Coker}\left(\mathcal{F} \otimes L_{y \mu}\right)$. For a given

$$
W \in \operatorname{Irr}^{\mathfrak{g}}(\operatorname{Coker}(\mathcal{F} \otimes \bar{N})),
$$

we define the related weight $\zeta_{W} \in \mathfrak{h}^{*}$ by $L_{\zeta_{W}} \cong \boldsymbol{\Xi}(W)$. The next statement describes the $\mathfrak{g}$-rough structure of simple $\tilde{\mathfrak{g}}$-supermodules in terms of the combinatorics of category $\mathcal{O}$.

Theorem 45 (Rough structure of simple $\tilde{\mathfrak{g}}$-supermodules) For any $\tilde{\mathfrak{g}}$-supermodule $S \in$ $\operatorname{Irr}{ }^{\widetilde{\mathfrak{g}}}(\operatorname{Coker}(\widetilde{\mathcal{F}} \otimes \operatorname{Ind} \bar{N}))$ and any $\mathfrak{g}$-supermodule $W \in \operatorname{Irr}^{\mathfrak{g}}(\operatorname{Coker}(\mathcal{F} \otimes \bar{N}))$, we have

$$
[\operatorname{Res}(S): W]=\left[\operatorname{Res}\left(\widetilde{L}_{S}\right): L_{\zeta_{W}}\right]
$$

Proof Equality (7.5) is obtained by applying $\boldsymbol{\Xi}$ to the left hand side and then using Lemma 44, cf. [38, Theorem 73]. 


\subsection{Classification of simple $\mathfrak{\mathfrak { g }}$-supermodules}

Theorem 39 reduces the problem of classification of all simple $\mathfrak{g}$-modules to the following problem. Let $\mathcal{I}$ denote the set of all primitive ideals in $U$ which appear as annihilators of all modules of the form $\bar{N}$, as chosen before (7.1). For each fixed simple $\mathfrak{g}$-module $S$ with annihilator $I \in \mathcal{I}$ (which we view as some $\bar{N}$ ), we have the corresponding bijection

$$
\boldsymbol{\Xi}(S): \operatorname{Irr}^{\mathfrak{g}}(\operatorname{Coker}(\mathcal{F} \otimes S)) \rightarrow \operatorname{Irr}^{\mathfrak{g}}\left(\operatorname{Coker}\left(\mathcal{F} \otimes L_{y \mu}\right)\right) .
$$

For $I \in \mathcal{I}$, let $\operatorname{Irr}^{\mathfrak{g}}(I)$ denote the set of isomorphism classes of simple $\mathfrak{g}$-modules whose annihilator is $I$. Consequently, we have:

Corollary 46 The set

$$
\coprod_{I \in \mathcal{I}} \coprod_{S \in \operatorname{Irr}^{\mathfrak{g}}(I)} \boldsymbol{\Xi}(S)^{-1}\left(\operatorname{Irr}^{\mathfrak{g}}\left(\operatorname{Coker}\left(\mathcal{F} \otimes L_{y \mu}\right)\right)\right)
$$

coincides with the set of isomorphism classes of simple $\mathfrak{g}$-modules.

This reduces, in some sense, the problem of classification of all $\mathfrak{g}$-modules to that of classification of all $\mathfrak{g}$-modules with annihilators in $\mathcal{I}$. For Lie algebras, this might look unimpressive. However, a similar reasoning applied to Corollary 41 gives the following very surprising extension of this to $\tilde{\mathfrak{g}}$ which, in the same sense, reduces the problem of classification of all $\tilde{\mathfrak{g}}$-supermodules to that of classification of all $\mathfrak{g}$-modules with annihilators in $\mathcal{I}$. Here, again, for each fixed simple $\mathfrak{g}$-module $S$ with annihilator $I \in \mathcal{I}$, we have the bijection

$$
\widetilde{\Xi}(S): \operatorname{Irr}^{\tilde{\mathfrak{g}}}(\operatorname{Coker}(\widetilde{\mathcal{F}} \otimes \operatorname{Ind}(S))) \rightarrow \operatorname{Irr}^{\tilde{\mathfrak{g}}}\left(\operatorname{Coker}\left(\widetilde{\mathcal{F}} \otimes \operatorname{Ind}\left(L_{y \mu}\right)\right)\right) .
$$

Theorem 47 (Classification of simple supermodules) The set

$$
\coprod_{I \in \mathcal{I}} \coprod_{S \in \operatorname{Irr}^{\mathfrak{g}}(I)} \operatorname{Irr}^{\tilde{\mathfrak{g}}}(\operatorname{Coker}(\tilde{\mathcal{F}} \otimes \operatorname{Ind}(S)))
$$

coincides with the set of isomorphism classes of simple $\tilde{\mathfrak{g}}$-supermodules.

Proof As $\mathfrak{g}$ is of type $A$, we can combine Theorem 23 with Proposition 22 to conclude, using Corollary 41 , that every simple $\tilde{\mathfrak{g}}$-supermodule appears in the right hand side of (7.6). The claim follows.

Although Theorem 47 is not as nice as [10, Theorem 4.1] (the latter result reduces classification of simple supermodules over basic classical Lie superalgebras of type I to classification of simple modules over the corresponding even part Lie algebra), it is fairly clear that [10, Theorem 4.1] does not extend to, for example, Q-type Lie superalgebras given in Subsection 7.6 in any easy way. At the same time, the set

$$
\operatorname{Irr}^{\tilde{\mathfrak{g}}}\left(\operatorname{Coker}\left(\tilde{\mathcal{F}} \otimes \operatorname{Ind}\left(L_{y \mu}\right)\right)\right)
$$

for Q-type Lie superalgebras can be described using Sect. 7.6. Therefore for Q-type Lie superalgebras, Theorem 47 provides significant progress in classification of simple supermodules. 


\subsection{Applications}

We give a short overview of Lie superalgebras to which our results are applicable and cannot be dealt with using the theory of [10]. We focus on $D(2,1 ; \alpha), F(4), Q(n)$ and generalized Takiff superalgebras.

First we quickly review the classification of simple modules in $\widetilde{\mathcal{O}}$. Let $\tilde{\mathfrak{g}}$ be a classical Lie superalgebra and choose a Cartan subalgebra $\mathfrak{a}$ of the derived Lie algebra $\mathfrak{g}^{\prime}=[\mathfrak{g}, \mathfrak{g}]$ of the underlying Lie algebra. Consider a Weyl group invariant bilinear form $\langle\cdot, \cdot\rangle$ on $\mathfrak{a}^{*}$. Following $[35, \S 2.4]$, we choose a $\omega \in \mathbb{R} \Phi$, where $\Phi$ is the set of roots of $\mathfrak{g}^{\prime}$, which is generic in the sense that $\langle\omega, \alpha\rangle \neq 0$ for all $\alpha \in \Phi$. Then we define a triangular decomposition

$$
\widetilde{\mathfrak{g}}=\tilde{\mathfrak{n}}^{-} \oplus \tilde{\mathfrak{h}} \oplus \tilde{\mathfrak{n}}^{+},
$$

where $\widetilde{\mathfrak{h}}$ is the direct sum of $\mathfrak{a}$-weight spaces corresponding to weights $\lambda$ satisfying $\langle\lambda, \omega\rangle=0$ and $\widetilde{\mathfrak{n}}^{ \pm}$corresponds to weights $\lambda$ with $\pm\langle\lambda, \omega\rangle>0$. Clearly $\mathfrak{h}:=\widetilde{\mathfrak{h}}_{\overline{0}}$ and $\mathfrak{n}^{ \pm}:=\widetilde{\mathfrak{n}}_{\overline{0}}^{ \pm}$give a triangular decomposition of $\mathfrak{g}$.

Assume now that $\left[\widetilde{\mathfrak{h}}_{\overline{1}}, \widetilde{\mathfrak{h}}_{\overline{1}}\right]=0$. Then simple $\widetilde{\mathfrak{h}}$-modules are the one dimensional $\mathfrak{h}$-modules with trivial $\widetilde{\mathfrak{h}}_{\overline{1}}$-action $\mathbb{C}_{\lambda}$, with $\lambda \in \mathfrak{h}^{*}$. We have the corresponding Verma module

$$
\widetilde{\Delta}_{\lambda}:=U(\widetilde{\mathfrak{g}}) \otimes_{U(\widetilde{\mathfrak{b}})} \mathbb{C}_{\lambda},
$$

with $\widetilde{\mathfrak{b}}=\widetilde{\mathfrak{h}} \oplus \widetilde{\mathfrak{n}}^{+}$acting on $\mathbb{C}_{\lambda}$ with trivial $\widetilde{\mathfrak{n}}^{+}$-action. It follows as in the classical case that $\widetilde{\Delta}_{\lambda}$ has a unique maximal submodule. We denote the quotient by $\widetilde{L}_{\lambda}$. The following lemma is well-known, see e.g. [35, Proposition 2].

Lemma 48 The set $\left\{\widetilde{L}_{\lambda}, \Pi \widetilde{L}_{\lambda} \mid \lambda \in \mathfrak{h}^{*}\right\}$ is a complete and irredundant set of representatives of isomorphism classes of simple objects in $\mathcal{O}$.

The one parameter family of simple Lie superalgebras $D(2,1 \mid \alpha)$, see [40, Chapter 4], satisfies the above assumptions and has underlying Lie algebra of type $A$. The multiplicities in $\operatorname{Res}\left(\widetilde{L}_{\lambda}\right)$ for $D(2,1 \mid \alpha)$ have been determined in [13]. Therefore our results provide a concrete approach to the rough structure problem. The simple Lie superalgebra $F(4)$, see [40, Chapter 4], has underlying Lie algebra $\mathfrak{s o}_{7} \oplus \mathfrak{s l}_{2}$. Based on Remark 25 we can thus expect to apply some of our results to this case. Note that there are also many examples of algebra $\tilde{\mathfrak{g}}$ as above which are far from simple, see for instance the generalized Takiff superalgebras introduced in [23, Section 3.3].

For Lie superalgebras of type $Q$, see [12,40], the statement in Lemma 48 has to be adapted, in particular, as some simple highest weight supermodules in type $Q$ are invariant under the parity change. However, an explicit classification of simple highest weight modules is known, see e.g., [12, Section 1.5.4] and [11, Lemma 2.1]. The category $\widetilde{\mathcal{O}}$ for type $Q$ Lie superalgebras has been intensively studied in e.g. [6,7,18,19]. However, the results in type $Q$ are not yet as complete as, say, for $\mathfrak{g l}(m \mid n)$. We conclude this subsection with more detailed analysis of two small rank examples for type $Q$ superalgebras:

Example 49 For the Lie superalgebra $\mathfrak{q}_{1}$, we have that $U\left(\mathfrak{q}_{1}\right)$ is isomorphic to $\mathbb{C}[x]$, where $x$ is odd. In particular, each simple supermodule is finite dimensional and thus belongs to $\widetilde{\mathcal{O}}$. The set $\mathcal{I}$ is a singleton and we can choose it to consist of any fixed primitive ideal $I$. The set $\operatorname{Irr}^{\mathfrak{g}}(I)$ is also a singleton. In this case the classification provided by Theorem 46 is tautological.

Example 50 For the Lie superalgebra $\mathfrak{q}_{2}$, a classification of simple supermodules can be found in [36] where it is given in a slightly different language and is much more detailed. 
Namely, [36] defines a correspondence between primitive ideals of $U\left(\mathfrak{q}_{2}\right)$ and those of $U\left(\mathfrak{g l}_{2}\right)$ and constructs explicit $U\left(\mathfrak{q}_{2}\right)-U\left(\mathfrak{g l}_{2}\right)$-bimodules, tensoring with which provides a bijection between simple $U\left(\mathfrak{g l}_{2}\right)$-modules and simple $U\left(\mathfrak{q}_{2}\right)$-supermodules with the annihilators connected by the defined correspondence. The description in Theorem 46 can be obtained from the description in [36] by putting together the classification pieces from [36] for primitive ideals connected by tensoring with finite dimensional (super)modules. In the $\mathfrak{q}_{2}$ case, there are two essentially different types of primitive ideals: those of finite codimension, for which Theorem 46 is tautological, just as in Example 49; and those of infinite codimension. In the first case, each $\operatorname{Irr}^{\mathfrak{g}}(I)$ is a singleton. In the second case, a classification of the elements in each $\operatorname{Irr}^{\mathfrak{g}}(I)$ can be obtained from the results of [5]. The set $\mathcal{I}$ can be chosen to consist of the annihilator of the trivial module, the annihilator of one fixed singular Verma module and the annihilator of one fixed simple Verma module in each weight lattice that does not contain any singular Verma modules.

\section{Kac functor preserves finite type socle and finite type radical for Lie superalgebras of type I}

In this section we prove the following observation which naturally connects to various previous parts of the paper. To state the most general result we work in the category $\tilde{\mathfrak{g}}$-sMod of all supermodules.

Theorem 51 Let $\widetilde{\mathfrak{g}}=\widetilde{\mathfrak{g}}_{-1} \oplus \widetilde{\mathfrak{g}}_{0} \oplus \tilde{\mathfrak{g}}_{1}$ be a Lie superalgebra of type I with $\mathfrak{g}:=\widetilde{\mathfrak{g}}_{0}$ and $\mathfrak{p}:=\tilde{\mathfrak{g}}_{0} \oplus \tilde{\mathfrak{g}}_{1}$. Then the corresponding Kac functor

$$
\mathrm{K}:=\operatorname{Ind}_{\mathfrak{p}}^{\widetilde{\mathfrak{g}}}: \mathfrak{g} \text {-sMod } \rightarrow \widetilde{\mathfrak{g}} \text {-sMod }
$$

(where $\tilde{\mathfrak{g}}_{1}$ acts trivially on $\mathfrak{g}$-modules) sends modules with finite type socle (resp radical) to modules with finite type socle (resp. radical) preserving the length of the socle (resp. radical).

Proof We start with the claim about the socle. Let $V \in \mathfrak{g}$-sMod be a module with finite type socle. Set $d:=\operatorname{dim} \widetilde{\mathfrak{g}}_{-1}$. Any $\widetilde{\mathfrak{g}}$-submodule of $\mathrm{K}(V)$ intersects $\Lambda^{d} \widetilde{\mathfrak{g}}_{-1} \otimes V$, see [10, Lemma 3.1]. The space $\Lambda^{d} \widetilde{\mathfrak{g}}_{-1} \otimes S$, with $S$ a simple submodule of $V$, therefore generates a simple $\widetilde{\mathfrak{g}}$-module of $K(V)$ and moreover intersects $\Lambda^{d} \widetilde{\mathfrak{g}}_{-1} \otimes V$ precisely in $\Lambda^{d} \widetilde{\mathfrak{g}}_{-1} \otimes S$. This follows from considering $K(V)$ as a $\mathbb{Z}$-graded module where elements of $\mathfrak{g}_{ \pm 1}$ act as operators in degree \pm 1 . The submodule generated by $\Lambda^{d} \widetilde{\mathfrak{g}}_{-1} \otimes \operatorname{soc}(V)$ is thus semisimple, essential and has same length as $\operatorname{soc}(V)$.

We proceed with the claim about radical. By Remark 5(i) and (iii) it follows that $K$ sends modules with finite type radical to modules with superfluous radical. For an arbitrary $\mathfrak{g}$-module $M$ and $\widetilde{\mathfrak{g}}$-module $N$, we have

$$
\operatorname{Hom}_{\mathfrak{g}}(K(M), N) \cong \operatorname{Hom}_{\mathfrak{g}}\left(M, N^{\widetilde{\mathfrak{g}}_{1}}\right) \text { with } \quad N^{\widetilde{\mathfrak{g}}_{1}}=\left\{v \in N: \widetilde{\mathfrak{g}}_{1} v=0\right\} .
$$

The above equation and [10, Theorem 4.1(i)] imply that $S_{L}:=L^{\widetilde{\mathfrak{g}}_{1}}$ is a simple $\mathfrak{g}$-module for each simple $\widetilde{\mathfrak{g}}$-module $L$, and the map $L \mapsto S_{L}$ induces a bijection of isomorphism classes of simple modules. For an arbitrary $\mathfrak{g}$-module $M$ and a simple $\tilde{\mathfrak{g}}$-module $L$ we thus have

$$
\operatorname{Hom}_{\mathfrak{g}}(K(M), L) \cong \operatorname{Hom}_{\mathfrak{g}}\left(M, S_{L}\right) .
$$

Consequently, if $M$ has finite type radical, the length of the top of $M$ and $K(M)$ coincide.

As an immediate corollary, we obtain the following. We call a Borel subalgebra $\tilde{\mathfrak{b}}$ of $\tilde{\mathfrak{g}}$ distinguished if it is of the form $\mathfrak{b} \oplus \mathfrak{g}_{1}$, for a Borel subalgebra $\mathfrak{b}$ of $\mathfrak{g}$. 
Corollary 52 All Verma supermodules with respect to distinguished Borel subalgebras over all Lie superalgebras of type I have simple socle.

Proof Verma supermodules over Lie superalgebras of type I can be obtained from Verma modules over Lie algebras using Kac functor. Therefore the claim follows from Theorem 51 and [16, Proposition 7.6.3(i)].

Acknowledgements Open access funding provided by Uppsala University. Chih-Whi Chen is partially supported by Vergstiftelsen. Kevin Coulembier is supported by the Australian Research Council. Volodymyr Mazorchuk is partially supported by the Swedish Research Council and the Göran Gustafsson Foundation.

Open Access This article is licensed under a Creative Commons Attribution 4.0 International License, which permits use, sharing, adaptation, distribution and reproduction in any medium or format, as long as you give appropriate credit to the original author(s) and the source, provide a link to the Creative Commons licence, and indicate if changes were made. The images or other third party material in this article are included in the article's Creative Commons licence, unless indicated otherwise in a credit line to the material. If material is not included in the article's Creative Commons licence and your intended use is not permitted by statutory regulation or exceeds the permitted use, you will need to obtain permission directly from the copyright holder. To view a copy of this licence, visit http://creativecommons.org/licenses/by/4.0/.

\section{References}

1. Beilinson, A., Bernštein, I.N.: Localisation de g-modules. C. R. Acad. Sci. Paris Sér. I Math. 292(1), 15-18 (1981)

2. Bell, A., Farnsteiner, R.: On the theory of Frobenius extensions and its application to Lie superalgebras. Trans. Am. Math. Soc. 335(1), 407-424 (1993)

3. Bernstein, J., Gelfand, S.: Tensor products of finite- and infinite-dimensional representations of semisimple Lie algebras. Compos. Math. 41(2), 245-285 (1980)

4. Bernstein, I., Gelfand, I., Gelfand, S.: A certain category of $\mathfrak{g}$-supermodules. Funkc. Anal. i Priloz. 10, 1-8 (1976)

5. Block, R.: The irreducible representations of the Lie algebra $\mathfrak{s l}(2)$ and of the Weyl algebra. Adv. Math. 39, 69-110 (1981)

6. Brundan, J.: Kazhdan-Lusztig polynomials and character formulae for the Lie superalgebra $\mathfrak{q}(n)$. Adv. Math. 182(1), 28-77 (2004)

7. Brundan, J., Davidson, N.: Type A blocks of super category O O. J. Algebra 473, 447-480 (2017)

8. Brylinski, J., Kashiwara, M.: Kazhdan-Lusztig conjecture and holonomic systems. Invent. Math. 64(3), 387-410 (1981)

9. Chen, C.-W., Coulembier, K.: The primitive spectrum and category $\mathcal{O}$ for the periplectic Lie superalgebra. Can. J. Math. arXiv:1805.08885

10. Chen, C.-W., Mazorchuk, V.: Simple supermodules over Lie superalgebras. arXiv:1801.00654

11. Chen, C.-W.: Reduction method for representations of queer Lie superalgebras. J. Math. Phys. 57, 051703 (2016)

12. Cheng, S.-J., Wang, W.: Dualities and Representations of Lie Superalgebras. Graduate Studies in Mathematics, vol. 144. American Mathematical Society, Providence (2012)

13. Cheng, S.-J., Wang, W.: Character formulae in category $\mathcal{O}$ for exceptional Lie superalgebras $D(2 \mid 1 ; \zeta)$. Transform. Groups 24(3), 781-821 (2019)

14. Cheng, S.-J., Mazorchuk, V., Wang, W.: Equivalence of blocks for the general linear Lie superalgebra. Lett. Math. Phys. 103(12), 1313-1327 (2013)

15. Coulembier, K.: The primitive spectrum of a basic classical lie superalgebra. Commun. Math. Phys. 348(2), 579-602 (2016)

16. Dixmier, J.: Enveloping Algebras. Graduate Studies in Mathematics, vol. 11. American Mathematical Society, Providence (1996)

17. Duflo, M.: Sur la classification des idéaux primitifs dans l'algèbre enveloppante d'une algèbre de Lie semi-simple. Ann. Math. (2) 105(1), 107-120 (1977)

18. Frisk, A.: Typical blocks of the category $\mathcal{O}$ for the queer Lie superalgebra. J. Algebra Appl. 6(5), 731-778 (2007) 
19. Frisk, A., Mazorchuk, V.: Volodymyr regular strongly typical blocks of $\mathcal{O}_{\mathfrak{q}}$. Commun. Math. Phys. 291(2), 533-542 (2009)

20. Geck, M.: Kazhdan-Lusztig cells and the Murphy basis. Proc. Lond. Math. Soc. (3) 93(3), 635-665 (2006)

21. Gorelik, M.: On the ghost centre of Lie superalgebra. Ann. Inst. Fourier (Grenoble) 50(6), 1745-1764 (2000)

22. Gorelik, M.: Annihilation theorem and separation theorem for basic classical Lie superalgebras. J. Am. Math. Soc. 15(1), 113-165 (2002)

23. Greenstein, J., Mazorchuk, V.: Koszul duality for semidirect products and generalized Takiff algebras. Algebr. Represent. Theory 20(3), 675-694 (2017)

24. Humphreys, J.: Representations of Semisimple Lie Algebras in the BGG Category $\mathcal{O}$. Graduate Studies in Mathematics, vol. 94. American Mathematical Society, Providence (2008)

25. Irving, R.: A filtered category $\mathcal{O}_{S}$ and applications. Mem. Am. Math. Soc. 83, 419 (1990)

26. Irving, R., Shelton, B.: Loewy series and simple projective modules in the category $\mathcal{O}_{S}$. Pac. J. Math. 132(2), 319-342 (1988)

27. Jantzen, J.: Einhüllende Algebren halbeinfacher Lie-Algebren. Ergebnisse der Mathematik und ihrer Grenzgebiete (3), vol. 3. Springer, Berlin (1983)

28. Joseph, A.: Kostant's problem, Goldie rank and the Gelfand-Kirillov conjecture. Invent. Math. 56(3), 191-213 (1980)

29. Kazhdan, D., Lusztig, G.: Representations of Coxeter groups and Hecke algebras. Invent. Math. 53(2), 165-184 (1979)

30. Khomenko, O., Mazorchuk, V.: Structure of modules induced from simple modules with minimal annihilator. Can. J. Math. 56(2), 293-309 (2004)

31. Knapp, A.W.: Lie Groups, Lie Algebras, and Cohomology. Mathematical Notes, vol. 34. Princeton University Press, Princeton (1988)

32. Kostant, B.: On the tensor product of a finite and an infinite dimensional representation. J. Funct. Anal. 20(4), 257-285 (1975)

33. Krause, G., Lenagan, T.: Growth of Algebras and Gelfand-Kirillov Dimension. Graduate Studies in Mathematics, vol. 22. American Mathematical Society, Providence (2000)

34. Mackaay, M., Mazorchuk, V., Miemietz, V., Zhang, X.: Analogues of centralizer subalgebras for fiat 2-categories and their 2-representations. J. Inst. Math. Jussieu. arXiv:1802.02078

35. Mazorchuk, V.: Parabolic Category $\mathcal{O}$ for Classical Lie Superalgebras. Advances in Lie Superalgebras, Springer INdAM Ser., vol. 7, pp. 149-166. Springer, Cham (2014)

36. Mazorchuk, V.: Classification of simple $\mathfrak{q}_{2}$-supermodules. Tohoku Math. J. (2) 62(3), 401-426 (2010)

37. Mazorchuk, V., Miemietz, V.: Serre functors for Lie algebras and superalgebras. Ann. Inst. Fourier 62(1), 47-75 (2012)

38. Mazorchuk, V., Stroppel, C.: Categorification of (induced) cell modules and the rough structure of generalised Verma modules. Adv. Math. 219(4), 1363-1426 (2008)

39. Miličić, D., Soergel, W.: The composition series of modules induced from Whittaker modules. Comment. Math. Helv. 72(4), 503-520 (1997)

40. Musson, I.: Lie Superalgebras and Enveloping Algebras. Graduate Studies in Mathematics, vol. 131. American Mathematical Society, Providence (2012)

41. Soergel, W.: The combinatorics of Harish-Chandra bimodules. J. Reine Angew. Math. 429, 49-74 (1992)

42. Stafford, J.: Nonholonomic modules over Weyl algebras and enveloping algebras. Invent. Math. 79(3), 619-638 (1985)

Publisher's Note Springer Nature remains neutral with regard to jurisdictional claims in published maps and institutional affiliations. 